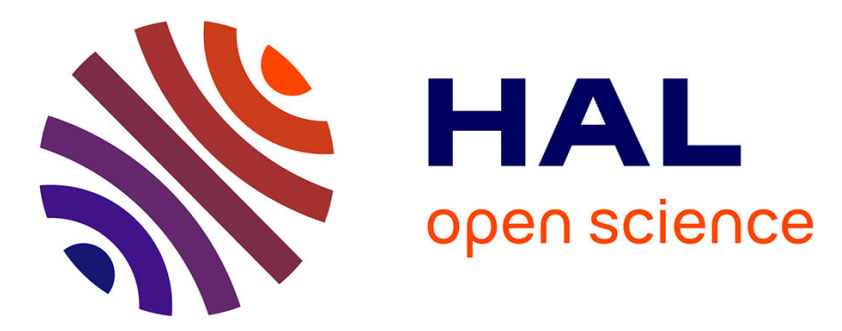

\title{
Revisited analysis of gas convection and heat transfer in micro channels: influence of viscous stress power at wall on Nusselt number
}

Xavier Nicolas, Eric Chénier, Chahinez Tchekiken, Guy Lauriat

\section{- To cite this version:}

Xavier Nicolas, Eric Chénier, Chahinez Tchekiken, Guy Lauriat. Revisited analysis of gas convection and heat transfer in micro channels: influence of viscous stress power at wall on Nusselt number. International Journal of Thermal Sciences, 2018, 134, pp.565-584. 10.1016/j.ijthermalsci.2018.05.049 . hal-01808802

\section{HAL Id: hal-01808802 \\ https://hal.science/hal-01808802}

Submitted on 6 Jun 2018

HAL is a multi-disciplinary open access archive for the deposit and dissemination of scientific research documents, whether they are published or not. The documents may come from teaching and research institutions in France or abroad, or from public or private research centers.
L'archive ouverte pluridisciplinaire HAL, est destinée au dépôt et à la diffusion de documents scientifiques de niveau recherche, publiés ou non, émanant des établissements d'enseignement et de recherche français ou étrangers, des laboratoires publics ou privés. 


\title{
Revisited analysis of gas convection and heat transfer in micro channels: influence of viscous stress power at wall on Nusselt number
}

\author{
Xavier NICOLAS, Eric CHENIER, Chahinez TCHEKIKEN, Guy LAURIAT \\ Université Paris-Est Marne-la-Vallée, MSME UMR 8208 CNRS, \\ 5 boulevard Descartes, 77454 Marne-la-Vallée Cedex 2, France
}

\begin{abstract}
This paper deals with the modeling of weakly rarefied and dilute gas flows in micro channels by the continuum approach, valid for Knudsen numbers smaller than about 0.1. It particularly focuses on the modeling of the associated heat transfer. The models proposed in the literature for the forced convection of gas flows in long micro channels between two infinite plates are more specifically discussed. The complete model for such flows is reminded after a compilation and a brief description of their possible applications in industries. The compatibility of the pressure work and viscous dissipation in the energy equation with the power of the viscous stress at the walls is discussed in detail. A dimensional analysis is proposed in the context of long micro channels. Analytical solutions for the velocity and temperature fields and for the Nusselt number are provided in the case of compressible micro-flows in isothermally heated flat plate channels, with pressure work and viscous dissipation included. The choice of an appropriate Nusselt number, including the power of the viscous stress at the wall, is particularly discussed. It is shown analytically and numerically, by solving the complete model for an isothermal wall micro-channel, that the Nusselt number tends to zero when the hydraulic diameter decreases, that is when the Reynolds number decreases and the Knudsen number increases. This could theoretically explain the very small values of the Nusselt number obtained in the experiments by Demsis et al. (2009, 2010).
\end{abstract}

Keywords: micro-channel, first-order slip flow, compressible flow, heat transfer, Nusselt number, analytical solution

Highlights: (maximum 85 characters including spaces per bullet point)

- Isothermally heated gas slipping flows in long plane micro-channels are considered

- Analytical solution of flow and heat transfer established from an asymptotic analysis

- Viscous stress power at the wall must be included in the total wall heat flux

- Wall Nusselt number tends to zero or to very small values for this flow type

- The very small values of the Nusselt number obtained in experiments are explained 


\section{Introduction}

Due to the increasing development of Micro Electro Mechanical Systems (MEMS), the study of liquid or gas flows and heat transfer in ducts, heated or not, whose hydraulic diameter, $D_{h}$, is of the order of a few microns (say 1 to $100 \mu \mathrm{m}$ ), has given rise to a considerable amount of works over the past twenty years. A recent review by Kandlikar et al. [1] is dedicated to them. It is shown that monophasic liquid flows in micro channels have a behavior similar to that observed at the macroscopic scale and the classical continuum mechanics model can be used (Navier-Stokes equations with no slip boundary conditions).

However, for gas flows at the microscopic scale, specific phenomena are observed and require appropriate models $[2,3]$. A slightly rarefied flow regime close to the wall, generated by the interaction between the gas molecules and the wall atoms, must be taken into account at the microscopic scale whereas it is negligible at the macroscopic scale or for liquid micro-flows. More specifically, a Knudsen layer whose thickness is of the order of the mean free path of the gas molecules, $\lambda$, is formed closed to the wall. In this layer, the velocity magnitudes of the gas molecules considered individually are different at a fixed distance from the wall, due to their interactions with the wall. In other words, in this layer, the gas is in a state of local thermodynamic non-equilibrium which results in non-linear mean velocity profiles and relations between stress and strain rates. From the continuum mechanics point of view, at the micro channel scale, when the Knudsen number is such that $0.001<K n=\lambda / D_{h}<0.1$, these phenomena translate into a slip velocity and a temperature jump at the wall and, possibly, a gas flow driven by the tangential temperature gradient along the wall called "thermal creep" [4]. The consequences of these phenomena on the macroscopic quantities such as the mass flow rate, the friction factor, the bulk temperature and the wall heat flux can be significant [5] and must be taken into account in the modeling of the convective heat transfer in MEMS with gas flows. Indeed they may have antagonistic effects on the heat transfer.

Gas micro-flows, possibly with heat transfer, can be found in :

- micro heat exchangers for the cooling of electronic components or in chemistry [6,7],

- micro pumps and turbines, including the thermal transpiration-driven Knudsen pumps for vacuum pumping applications [8-10],

- micro-systems for the species separation in gas mixtures such as the method of gas separation by membranes [11],

- micro gas analyzers such as micro mass spectrometers and micro-chromatographs [12,13],

- supersonic gas flows in micro-nozzles to control the nano-satellite attitude or the boundary layers in aerodynamics [14-18],

- artificial lungs $[19,20]$,

- pressure, flow rate and temperature micro-sensors in gas flows [21,22], etc.

This paper investigates the theoretical models available to simulate and analyze the slightly rarefied gas micro-flows with heat transfer, when $K n \leqslant 0.1$. We focus on the modeling of forced convection of pure diluted gases in micro channels by a continuous approach based on the NavierStokes equations and first order slip and temperature jump boundary conditions. It appears that simplified models are often used in the literature for this flow type, but without relevant 
justification and with recurrent errors propagating from one paper to the other, particularly concerning the heat transfer analysis and the energy equation. Our purpose is to provide a consistent model for gas micro-flows and heat transfer and to compare with the vanishing values of the Nusselt number obtained in experiments [23,24].

In that aim, the characteristic length scales of the continuum description and the way of modeling the Knudsen layer are reminded in $\S 2$. The values of the slip and temperature jump coefficients are particularly discussed. The complete model for forced convection in heated micro channels is established and discussed in $§ 3$. A dimensional analysis is developed and the analytical solution of the temperature field given by a simplified asymptotic model for compressible gas convection in an isothermal micro-channel is established in $\S 4$. This solution is compared with the numerical solution of the full model obtained from finite volume simulations in $\S 5$. Furthermore, from the numerical simulations, the heat flux balances for slip and no slip flows and incompressible and compressible flows are analysed in details. The numerical method to solve the full model is presented in $§ 5.1$ and the analytical and numerical solutions are compared in $§ 5.2$. The heat flux balances and the very small values of the Nusselt number obtained in the experiments by Demsis et al. $[23,24]$ are explained in $§ 5.3$.

\section{Length scales of the continuum description and Knudsen layer modeling}

The mean free path, $\lambda$, is the average distance traveled by the molecules between two successive collisions. It is the main scale to evaluate the rarefaction rate in a gas flow and the validity domain of the continuum description. In this paper, the most standard definition used for ideal gases is retained [2, $5,26-28]$ :

$$
\lambda=\frac{\mu}{p} \sqrt{\frac{\pi r T}{2}}=\frac{\mu}{\rho} \sqrt{\frac{\pi}{2 r T}}=\mu \sqrt{\frac{\pi}{2 p \rho}}
$$

where $r$ is the specific gas constant.

A scale analysis of the breakdown of the continuum description of gas flows was presented by Bird [25] and recalled by Gad-El-Hak [2,29], Colin [30] and Zhang et al. [31]. For dilute gases, the limit on the range of validity of the continuum equations is first due to the local thermodynamic non-equilibrium and the presence of very steep gradients in the flow fields (on characteristic lengths, $L$, of the same order as the mean free path, $\lambda$ ), rather than due to statistical fluctuations of the macroscopic variables. It is generally admitted that the thermodynamic equilibrium is satisfied and the Navier-Stokes equations are valid everywhere for $K n=\lambda / D_{h}<0.001$ to 0.01 , and in the flow core only for $0.01<K n<0.1$. In the latter case, in the thin layer close to the wall (the Knudsen layer), the continuum equations are not valid because the gas molecules only "see" a half-space where the nature of the shocks with the wall is different from the inter-molecular shocks: a local thermodynamic non-equilibrium is present in this zone.

An accurate description of the thin Knudsen layer whose thickness is between about $\lambda$ and $3 \lambda[5,31]$ is crucial for microfluidics applications. Momentum and energy are indeed transferred between the gas and the wall through this layer. An ill description has thus significant consequences on the evaluations of the mass flow rate and friction factor, or on the maximum bulk 
temperature and wall heat flux. For $0.001<K n<0.1$, a continuous approach coupled with a modeling of the flow and heat transfer in the Knudsen layer is generally considered. The most used model consists in solving the Navier-Stokes equations with slip boundary conditions and semi-empirical coefficients, such as the "accommodation coefficient" or the "slip length", to model the gas/wall interaction. A similar model is used for heat transfer: the energy equation is solved with a temperature jump boundary condition to mimic the thermal resistance of the Knudsen layer. The choice of the slip and temperature jump coefficients is detailed below because inadaquate or unconsistent values of these coefficients are regularly used in the literature.

\subsection{Slip and thermal creep boundary conditions}

The first-order slip boundary condition was first introduced by Navier in 1823 [36], then independently by Maxwell in 1879 [37]. The simplified form on an impermeable wall writes:

$$
\begin{aligned}
u_{g}-u_{w} & =\left.L_{s} \frac{\partial u}{\partial \vec{n}}\right|_{g} \\
v_{g} & =0
\end{aligned}
$$

where $u$ and $v$ are the velocity components of the gas, tangential and normal to the wall respectively, $u_{g}$ is the slip velocity of the gas on the wall, $u_{w}$ is the velocity of the wall $\left(u_{w}=0\right.$ in general), $L_{s}$ is the slip length and $\vec{n}$ is the direction normal to the wall directed toward the gas with $\partial u / \partial \vec{n}=\nabla u \cdot \vec{n}$. Here and in the following, the subscript " $g$ " is used to denote quantities on the gas side of the wall (slip-related quantities associated with the gas molecules in contact with the wall) and the subscript " $w$ " is used to denote the quantities on the solid side of the wall.

The slip boundary condition (2) and the comparison of the "true" and modeled velocity profiles of the gas in the Knudsen layer are illustrated in the left part of Fig. 1. The "true" velocity profile (in red) is non-linear close to the wall and presents a slip speed at the wall denoted $u_{g, t r u e}$. The Navier and Maxwell model consists in approximating this velocity profile in the Knudsen layer by the blue profile whose slip velocity, $u_{g}$, is greater than the actual slip velocity, $u_{g, t r u e}$, by considering that the difference $u_{g}-u_{w}$ is proportional to the normal velocity gradient along the Knudsen layer, $\left.\frac{\partial u}{\partial \vec{n}}\right|_{g}$. The slip length, $L_{s}$, in this model is a semi empirical parameter, proportional to the mean free path, $\lambda$, and depends on the gas and wall nature, on the wall roughness and, more generally, on the type of gas/wall interaction (diffuse, specular or mixed specular and diffuse scattering of the gas molecules at the wall). $L_{s}$ must be evaluated so that $u_{g}$ provides a good approximation of the velocity profile outside the Knudsen layer.

There are many formulations of $L_{s}$ in the literature which are more or less equivalent: some formulations depend on a viscous slip coefficient, $\sigma_{\mu}$, such as $L_{s}=\sigma_{\mu} \lambda$; others are function of the Tangential Momentum Accommodation coefficient (TMAC) ${ }^{1}$, denoted $\sigma_{u}$ here, such as

\footnotetext{
${ }^{1}$ TMAC represents the average fraction of the momentum transferred to the wall atoms in the tangential direction by the incident gas molecules impacting the wall : $0<\sigma_{u}<1$ in case of partial accommodation of the gas molecules with the wall, $\sigma_{u}=1$ in case of a diffuse reflection (or full accommodation) of the gas molecules
} 


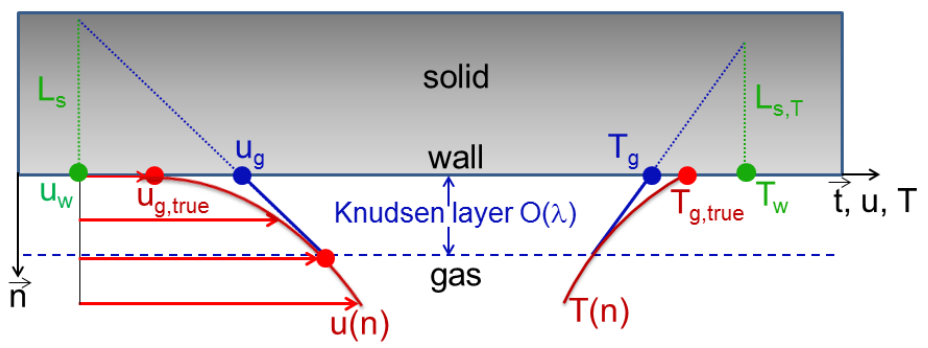

Fig. 1: Schematic representation of the true (in red) and approximated (in blue) velocity and temperature profiles in the Knudsen layer and (in green) of the slip length, $L_{s}$, and temperature jump length, $L_{s, T}$.

$L_{s}=\left[\varpi_{u}\left(2-\sigma_{u}\right) / \sigma_{u}\right] \lambda$ with $\varpi_{u}$ a corrective coefficient $[38,39]$. Recently, following the extensive work by Sharipov [4], the first formulation seems to settle as a standard [1,31]. However, the slip boundary condition given by equation (2) is very simplified. A more general form, valid for non isothermal flows, can be written :

$$
u_{g}-u_{w}=\left.\sigma_{\mu} \lambda\left(\frac{\partial u}{\partial \vec{n}}+\frac{\partial v}{\partial \vec{t}}\right)\right|_{g}+\left.\sigma_{\theta}\left(\frac{\mu r}{p} \frac{\partial T}{\partial \vec{t}}\right)\right|_{g}
$$

where $\sigma_{\mu}$ and $\sigma_{\theta}$ are the viscous and thermal slip coefficients (detailed below), $\mu$ the dynamic viscosity and $\vec{t}$ the direction tangential to the wall. Compared with Eq. (2), the slip velocity in Eq. (4) also depends on the tangential gradients of the normal velocity component, $v$, and temperature, $T$. The term $\left.\frac{\partial v}{\partial \vec{t}}\right|_{g}$ vanishes if the wall is plane and smooth. However, it must be taken into account in case of high roughness or sharp curvature of the wall, when the radius of curvature is about or less than the mean free path, $\lambda$, of the gas molecules [40,41].

The last term in Eq. (4) is the thermal creep or thermal transpiration term. It reflects the presence of a tangential flow along the wall, generated by a longitudinal temperature gradient. The thermal creep is directed from low temperatures to high temperatures. Thanks to a dimensional analysis, we will show that this phenomenon is only significant at very small Reynolds numbers. When the thermal creep is negligible, the first order slip boundary condition (4) on a plane and smooth wall simply writes:

$$
u_{g}-u_{w}=\left.\sigma_{\mu} \lambda \frac{\partial u}{\partial \vec{n}}\right|_{g}
$$

Sharipov [4] proposed a synthesis of the theoretical and experimental values of the viscous and thermal slip coefficients, $\sigma_{\mu}$ and $\sigma_{\theta}$, and also the thermal jump coefficient $\xi_{T}$ (see Eq. (6)) available in the literature. The theoretical data in [4] are mainly calculated from the Boltzmann kinetic equation or various kinetic equations, like the BGK kinetic model. Due to the different definitions of the mean free path $\lambda$ between [4] and Eq. (1), all the data for these coefficients in [4] must be multiplied by the factor $\frac{2}{\sqrt{\pi}}$ to be used with the present formulation. Thus, we get $\sigma_{\mu} \approx 1.1, \sigma_{\theta} \approx 1.2$ and $\xi_{T} \approx 2.2$ with the present definition of $\lambda$ (Eq. (1)), in the case of diffuse scattering (or full accommodation) of the gas molecules at the wall. To take into and $\sigma_{u} \rightarrow 0$ in case of specular reflection (no accommodation). 
account a non complete accommodation, it is generally assumed that a part, evaluated by the coefficient $T M A C=\sigma_{u}$, of incident particles is scattered diffusely, while the rest of particles, i.e. the $\left(1-\sigma_{u}\right)$ part, is reflected specularly. Thus, in case of non diffuse scattering, only a part of the molecules accommodates with the wall and it is shown that $\sigma_{\mu}$ is greater than 1.1 and diverges for a specular scattering (for $\sigma_{u} \rightarrow 0$ ), whereas $\sigma_{\theta}$ is smaller than 1.2 and tends to $\frac{3}{4} \times \frac{2}{\sqrt{\pi}}$ for a specular scattering. For the sake of simplicity and as, in most of the experiments, the gas/wall interaction is diffuse $\left(T M A C=\sigma_{u} \approx 1\right)$, we will take $\sigma_{\mu}=\sigma_{\theta}=1$ and $\xi_{T}=2$ in the present numerical applications. Note that, in most of the published numerical works, $\sigma_{\mu}=1$ and $\sigma_{\theta}=3 / 4$ are used at the same time what is erroneous since the former expression corresponds to a diffuse scattering and the latter to a specular scattering.

\subsection{Thermal jump boundary condition}

Similarly to the Navier and Maxwell slip boundary conditions, Smoluchowski (1898) [42] introduced an equivalent boundary conditions to characterize the temperature jump observed at the wall in the Knudsen layer. This temperature jump and its modeling are illustrated in the right part of Fig. 1. The boundary conditions equivalent to Eq. (5) but for the temperature can be written, at the first order with $K n[1,4,31]$ :

$$
T_{g}-T_{w}=\left.\xi_{T} \lambda \frac{\partial T}{\partial \vec{n}}\right|_{g}
$$

where $T_{g}$ is the gas temperature at the wall, $T_{w}$ the wall temperature and $\xi_{T}$ the temperature jump coefficient such as the thermal jump length in Fig. 1 is $L_{s, T}=\xi_{T} \lambda$. According to the bibliographical synthesis [4], with $\lambda$ given by Eq. (1), $\xi_{T} \approx 2.1$ to 2.2 for diffuse scattering of mono atomic gases ( $\mathrm{He}, \mathrm{Ar}, \mathrm{Ne}$ and $\mathrm{Xe}$ ) and their binary mixtures at the walls.

In the literature, Eq. (6) is often considered in the following form:

$$
T_{g}-T_{w}=\left.\frac{2-\sigma_{T}}{\sigma_{T}} \frac{2 \gamma}{\gamma+1} \frac{\lambda}{\operatorname{Pr}} \frac{\partial T}{\partial \vec{n}}\right|_{g}
$$

where $\sigma_{T}$ is the thermal accommodation coefficient [3] (equivalent of TMAC for the thermal energy), $\gamma=C_{p} / C_{v}$ is the isentropic coefficient and $\operatorname{Pr}$ the Prandtl number. However, in the case of a mono-atomic (respectively diatomic) ideal gas, $\gamma=5 / 3$ (respectively $7 / 5$ ) and $\operatorname{Pr} \approx 0.7$. So, as $\sigma_{T}=1$ for a diffuse gas/wall interaction, the factor $\frac{2-\sigma_{T}}{\sigma_{T}} \frac{2 \gamma}{\gamma+1} \frac{1}{P r}$ in Eq. (7) is approximately equal to 1.6 to 1.8 for bi and mono atomic gases which is noticeably smaller than $\xi_{T} \approx 2.1$ to 2.2 given by Sharipov [4].

Finally note that the temperature jump boundary condition that reflects the rarefaction and the presence of the Knudsen layer is valid and must be applied regardless of the type of thermal condition applied: fixed temperature, imposed heat flux or heat conduction in the wall. We insist on this point because some authors did not take into account the temperature jump condition when a heat flux is imposed on a wall to determine the wall temperature. 


\section{Mathematical model for gas flows in micro channels}

\subsection{Main physical phenomena and modeling issues}

For flows in micro channels of large aspect ratio, $L / D_{h}$, and typical sizes $1 \mu m \leq D_{h} \leq$ $10 \mu \mathrm{m}$ et $100 \mu \mathrm{m} \leq L \leq 1 \mathrm{~mm}$, submitted to a moderate heating of the walls and to pressure variations between the inlet and the outlet of the channel of the order of one bar to a few bars, the conversion of the mechanical work of the viscous forces into internal energy is very important. It can also be shown that the Mach number, $M a=\bar{u} / \sqrt{\gamma r T}$, and the Brinkman number, $B r=\mu \bar{u}^{2} /(k \Delta T)$, can reach or exceed the unit and the Reynolds number, $R e=\bar{u} D_{h} / \nu$, and the Péclet number, $P e=\bar{u} D_{h} / \alpha$, can vary between $10^{-2}$ and $10^{2}$. Accordingly, modeling this type of flow requires to take into account a wide range of physical phenomena:

- the local thermodynamic non equilibrium and rarefaction effects in the Knudsen layer as described in the previous sections (dynamic slip, temperature jump and thermal creep),

- the compressibility of the gas (the volume expansion and cooling of the gas associated with the pressure work) [43-46],

- the viscous dissipation (heat source) $[45,46]$,

- the work of the viscous forces at the wall in the presence of dynamic slip [27, 47, 48],

- the variation of the physical properties with temperature $[49,50]$,

- the dominant thermal and dynamical axial diffusions at the inlet and outlet boundaries when $R e$ and $P e$ are less than unity,

- the heat conduction in the walls (conjugate heat transfer) because they are usually thicker than the channel and more conducting than the gas [51].

- the relative roughness of the walls, which can be important at these small scales [31,52-56].

A bibliographical review of numerous numerical studies on this subject shows that all these effects are never taken into account simultaneously. This may be justified in some cases when the effect of the omitted term in the model is negligible on the dynamic and thermal behavior of the flow. However, the justification of the simplifying assumptions used in the model is often omitted. One objective of this paper is thus to study the influence of different phenomena described above in the case of gas flows in 2D straight micro channels. The analysis will particularly focus on the thermal aspects: we will analyze the influence of different terms in the energy equation and thermal boundary conditions on the evolution of the temperature field and Nusselt number.

To this end, we present below full and simplified mathematical models for modeling gas micro-flows in the framework of continuum mechanics. The general conservation equations are presented in $\S 3.2$. The viscous stress power at the walls is discussed in $\S 3.3$ and its influence on the sum of the pressure work and viscous dissipation in $§ 3.4$. The summary of the governing equations and boundary conditions in their dimensional form is given in $§ 3.5$. The dimensionless equations and the main results derived from a dimensional analysis of these micro flows are presented in $\S 3.6$. 


\subsection{General conservation equations}

In the present study, we consider steady gas flows in isothermal parallel-plate micro channels with the inlet and outlet pressures prescribed at $p_{\text {in }}$ and $p_{\text {out }}$. The geometry is presented in Fig. 2. The flow is assumed to be two-dimensional, symmetrical through the horizontal midplane, steady, compressible and laminar. The fluid is an ideal gas with potentially variable physical properties. The governing equations are the mass, momentum and energy conservation equations in which the body forces are neglected with respect to the viscous forces.

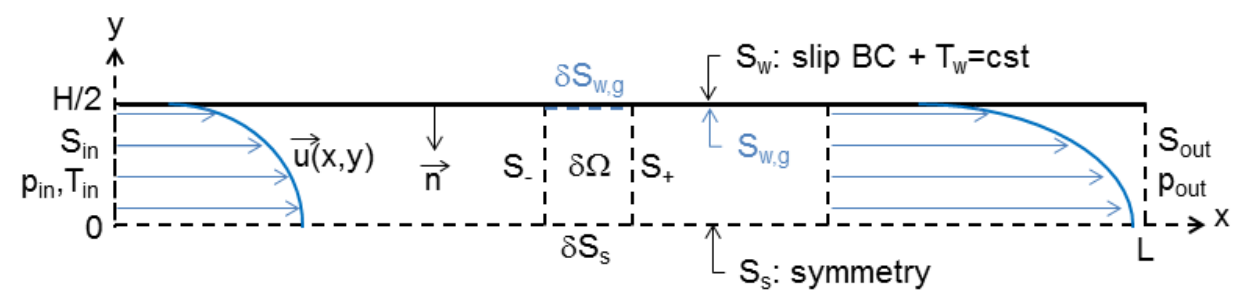

Fig. 2: Considered geometry for gas flows in 2D isothermal micro channels

The continuity and Navier-Stokes equations write [57]:

$$
\begin{gathered}
\nabla \cdot(\rho \vec{v})=0 \\
\nabla \cdot(\rho \vec{v} \otimes \vec{v})=-\nabla p+\nabla \cdot \overline{\bar{\tau}}
\end{gathered}
$$

where the shear stress tensor $\overline{\bar{\tau}}$ is defined for a Newtonian-Stokes fluid by :

$$
\overline{\bar{\tau}}=\mu\left(\nabla \vec{v}+\nabla \vec{v}^{t}\right)-\frac{2}{3} \mu \nabla \cdot \vec{v} \overline{\bar{I}}
$$

By noting $h$ the enthalpy and $e_{c}=\vec{v}^{2} / 2$ the kinetic energy per mass unit of the gas, the total energy conservation equation writes [57]:

$$
\nabla \cdot\left(\rho \vec{v} h+\rho \vec{v} e_{c}-k \nabla T-\overline{\bar{\tau}} \cdot \vec{v}\right)=0
$$

With the present assumptions, the mechanical energy balance (or equation of change for mechanical energy) can be written:

$$
\nabla \cdot\left(\rho \vec{v} e_{c}-\overline{\bar{\tau}} \cdot \vec{v}\right)=-\vec{v} \cdot \nabla p-\overline{\bar{\tau}}: \nabla \vec{v}
$$

By subtracting Eq. (12) from Eq. (11), we get the enthalpic form of the energy equation:

$$
\nabla \cdot(\rho \vec{v} h-k \nabla T)=\vec{v} \cdot \nabla p+\overline{\bar{\tau}}: \nabla \vec{v}
$$

The viscous dissipation $(V D)$ is a heat source term in the enthalpy equation $(\overline{\bar{\tau}}: \nabla \vec{v}>0)$ whereas the pressure work $(P W)$ term is a heat $\operatorname{sink}(\vec{v} . \nabla p<0)$ since the velocity vector, $\vec{v}$, and the pressure gradient, $\nabla p$, have opposite directions in a channel flow of constant section. For a compressible gas flow in a constant section duct, both the magnitudes of $V D$ and $P W$ increase downstream due to the gas expansion (acceleration) at the channel outlet and the 
transformation of the enthalpy/internal energy into kinetic energy.

For an ideal gas, the density is given by the equation of state:

$$
\rho(p, T)=\frac{p}{r T}
$$

where $r$ is the specific gas constant, and the enthalpy per unit of mass is computed by:

$$
h(T)-h_{r e f}\left(T_{r e f}\right)=\int_{T_{r e f}}^{T} C_{p}(T) d T
$$

where $h_{\text {ref }}=h\left(T_{\text {ref }}\right)$ is the known reference enthalpy at the reference temperature $T_{r e f}$. In the previous equations, the thermal conductivity, $k$, the dynamic viscosity, $\mu$, and the specific heat capacity at constant pressure, $C_{p}$, are a priori temperature dependent. However, in the present study, since the temperature variations are small due to the used isothermal wall condition, the temperature dependence of the physical properties is negligible and not considered hereafter.

\subsection{Viscous stress power $(V S P)$ at impermeable walls}

We consider a compressible, non-isothermal gas flow in a channel (as shown in Fig.2). The boundary of the flow domain, $\Omega$, is limited by the following four surfaces: the inlet section, $S_{i n}$, the outlet section, $S_{\text {out }}$, the surface on the gas side of the impermeable wall, $S_{w, g}$, and the symmetry surface, $S_{s}$. $\vec{n}$ is the inward normal unit vector of the closed surface $\Sigma=$ $S_{\text {in }} \cup S_{\text {out }} \cup S_{w, g} \cup S_{s}$ (Fig. 2). Then, by integrating the total energy equation (11) on $\Omega$, applying the Gauss theorem and simplifying, we get:

$$
\iint_{S_{\text {in }} \cup S_{\text {out }}}\left(\rho \vec{v} h+\rho \vec{v} e_{c}-k \nabla T-\overline{\bar{\tau}} \cdot \vec{v}\right) \cdot \vec{n} d S+\iint_{S_{w, g}}\left(-\left.k \nabla T\right|_{g}-\left.\overline{\bar{\tau}} \cdot \vec{v}\right|_{g}\right) \cdot \vec{n} d S=0
$$

in which the power of the viscous stress $\left.(\overline{\bar{\tau}} \cdot \vec{v}) \cdot \vec{n}\right|_{g} \neq 0$ in case of slipping flow along the wall since $\vec{v}_{g}=u_{g}(x) \overrightarrow{e_{x}} \neq \overrightarrow{0}$ and

$$
\left.(\overline{\bar{\tau}} \cdot \vec{v}) \cdot \vec{n}\right|_{g}=\left.\mu_{g} u_{g} \frac{\partial u}{\partial \vec{n}}\right|_{g}
$$

Therefore, in case of rarefaction and slip conditions, the conservation of the heat flux between the gas and the solid wall implies that the total heat flux density transmitted through the walls, $\vec{q}_{t, w} \cdot \vec{n}=q_{t, w}(x)\left[W / m^{2}\right]$, is equal to the sum of the diffusion heat flux and the power of the viscous stress:

$$
\vec{q}_{t, w} \cdot \vec{n}=\left(-\left.k \nabla T\right|_{g}-\left.\overline{\bar{\tau}} \cdot \vec{v}\right|_{g}\right) \cdot \vec{n}
$$

Thus, using Eq. (17), the total heat flux density transmitted through the wall writes:

$$
q_{t, w}=\underbrace{-\left.k_{g} \frac{\partial T}{\partial \vec{n}}\right|_{g}}_{\text {diffusion heat flux }}-\underbrace{\left.\mu_{g} u_{g} \frac{\partial u}{\partial \vec{n}}\right|_{g}}_{\text {viscous stress power } \geq 0}
$$

where $k_{g}(x)=k\left(T_{g}(x)\right)$ and $\mu_{g}(x)=\mu\left(T_{g}(x)\right)$ are the gas conductivity and viscosity at the gas 
temperature close to the walls, $T_{g}(x)$ (see Fig.1 and Eqs. (6) or (7)). In Eq. (19), the power of the viscous stress at the wall (the last term under the brace) is positive or null. Thus the total heat flux density through the wall, $q_{t, w}$, is always smaller than the diffused or convected heat flux density to the fluid and it can be positive or negative according to the relative magnitude of the two R.H.S terms.

The expression (19) of the wall heat flux was presumably introduced by Maslen (1958) [58] then discussed by Sparrow and Lin (1962) [27]. Since then, only a few authors have taken into account the power of the viscous stress at the wall in their analysis of heat transfer $[44,47,59]$. So, as most of the authors neglected this contribution, it was eventually forgotten. Hong and Asako [48] reiterated its importance in a paper in 2010 and, more recently, some papers have used total heat flux expressions similar to Eq. (19) to analyse heat transfer in isoflux micro channels $[60,61]$. In the present paper, we will analyze the contribution of $q_{t, w}$ on the Nusselt number, in the case of isothermal micro channels. We will show that it allows to recover very low values of the Nusselt number as it was experimentally obtained by Demsis et al. [23, 24].

It appears that to get a continuum model consistent with the conservation of the total heat flux, it is necessary to consider the power of the viscous stress at the wall when slip is present. Eq. (19) is therefore the consequence of the presence of a slipping flow and this condition is independent of the temperature jump condition, Eqs. (6) or (7). So, in case of moderate rarefaction $(0.001 \leq K n \leq 0.1)$, Eqs. (19) and (6) or (7) must be simultaneously satisfied in the continuum model. Most of the published works consider only one of these two conditions.

\subsection{Pressure work $(P W)$ and viscous dissipation $(V D)$ for weakly compressible flows}

In numerous studies on gas micro-flows, the contributions of the pressure work and the viscous dissipation in the energy equation (13) were either both neglected or the flow was considered quasi-incompressible and only the viscous dissipation was accounted for. We remind here some important theoretical results on these assumptions.

We consider first the integration of the mechanical energy equation (12) on the slice $\delta \Omega$ of the impermeable wall micro-channel of Fig. 2, whose boundary is $\delta \Sigma=S_{-} \cup S_{+} \cup \delta S_{w, g} \cup \delta S_{s}$ and $\vec{n}$ is the inward normal unit vector. After applying the Gauss theorem and simplifying, we get:

$$
\underbrace{\iiint_{\delta \Omega}(\vec{v} \cdot \nabla p+\overline{\bar{\tau}}: \nabla \vec{v}) d \Omega}_{P W+V D}=\underbrace{\int \underbrace{}_{V S P}-(\overline{\bar{\tau}} \cdot \vec{v}) \cdot \vec{n} d S}_{I_{S_{-} \cup S_{+} \approx 0} \iint_{S_{-} \cup S_{+}}\left(\rho \vec{v} e_{c}-\overline{\bar{\tau}} \cdot \vec{v}\right) \cdot \vec{n} d S}
$$

where the first R.H.S integral $I_{S_{-} \cup S_{+}}$is null for fully established incompressible flows and is negligible for weakly compressible flows since, in this case, the velocity profile is (nearly) the same on the inlet $\left(S_{-}\right)$and outlet $\left(S_{+}\right)$sections of $\delta \Omega$. Furthermore the last integral of the viscous stress power $(V S P)$ on the wall $\left(\delta S_{w, g}\right)$ vanishes for no slipping flows.

Consequently the integral, $P W+V D$, of the pressure work and viscous dissipation on the slice $\delta \Omega$ is very small (negligible) for fully established no slipping flows with weak streamwise 
density variations: in this case, the pressure work compensates the viscous dissipation, both locally (on a channel slice) and also on the whole domain, except at the inlet and the outlet where the entrance and expansion effects can be important. It appears thus that it is preferable to neglect $P W$ and $V D$ at the same time instead of one at a time. This is all the more true since the flow is compressible. This is perfectly illustrated in the numerical study of heated compressible flows in 2D micro channels by Hong et al. (2007) [45].

For established and moderately rarefied slipping flows $(0.001 \leq K n \leq 0.1)$ with small streamwise density variations, it appears from Eq. (20) that the first integral term in the RHS of Eq. (20) is approximately equal to zero. Therefore, $P W+V D \approx V S P$ : the sum of the pressure work and viscous dissipation does not vanish and is approximately equal to the power of the viscous stress at the wall. As a result, $P W+V D$ cannot be neglected in the energy equation if $V S P$ is not negligible at the wall and vice versa.

\subsection{Governing equations for two-dimensional flows of ideal gases in an isothermally heated flat-plate channel}

Referring to the coordinate system of Fig. 2, the governing equations and boundary conditions for two-dimensional flows of ideal gases in the $x$ direction, between two isothermal parallel smooth walls of length $L$, separated by a distance $H$ in the transverse $y$ direction, are summed up below. By denoting $(u, v)$ the velocity components in directions $(x, y)$ of the velocity vector $\vec{v}$, the equations (8), (9) and (13) can be written:

$$
\begin{gathered}
\frac{\partial \rho u}{\partial x}+\frac{\partial \rho v}{\partial y}=0 \\
\frac{\partial \rho u u}{\partial x}+\frac{\partial \rho u v}{\partial y}=-\frac{\partial p}{\partial x}+\frac{\partial}{\partial x}\left[2 \mu\left(\frac{\partial u}{\partial x}-\frac{1}{3}\left(\frac{\partial u}{\partial x}+\frac{\partial v}{\partial y}\right)\right)\right]+\frac{\partial}{\partial y}\left[\mu\left(\frac{\partial u}{\partial y}+\frac{\partial v}{\partial x}\right)\right] \\
\frac{\partial \rho u v}{\partial x}+\frac{\partial \rho v v}{\partial y}=-\frac{\partial p}{\partial y}+\frac{\partial}{\partial x}\left[\mu\left(\frac{\partial u}{\partial y}+\frac{\partial v}{\partial x}\right)\right]+\frac{\partial}{\partial y}\left[2 \mu\left(\frac{\partial v}{\partial y}-\frac{1}{3}\left(\frac{\partial u}{\partial x}+\frac{\partial v}{\partial y}\right)\right)\right] \\
C_{p}\left(\frac{\partial \rho u T}{\partial x}+\frac{\partial \rho v T}{\partial y}\right)=\left[\frac{\partial}{\partial x}\left(k \frac{\partial T}{\partial x}\right)+\frac{\partial}{\partial y}\left(k \frac{\partial T}{\partial y}\right)\right]+\left(u \frac{\partial p}{\partial x}+v \frac{\partial p}{\partial y}\right)+\overline{\bar{\tau}}: \nabla \vec{v}
\end{gathered}
$$

where the viscous dissipation writes:

$$
\overline{\bar{\tau}}: \nabla \vec{v}=\mu\left\{2\left[\left(\frac{\partial u}{\partial x}\right)^{2}+\left(\frac{\partial v}{\partial y}\right)^{2}\right]+\left(\frac{\partial u}{\partial y}+\frac{\partial v}{\partial x}\right)^{2}-\frac{2}{3}\left(\frac{\partial u}{\partial x}+\frac{\partial v}{\partial y}\right)^{2}\right\}
$$

The above set of equations is completed with the state equation (14) of the ideal gas.

At the inlet section $x=0$, uniform pressure and temperature are applied:

$$
p(0, y)=p_{\text {in }}, \quad \frac{\partial u}{\partial x}(0, y)=0, \quad v(0, y)=0, \quad T(0, y)=T_{\text {in }}
$$

At the outlet section $x=L$, the pressure is fixed and fully developed flow conditions are applied:

$$
p(L, y)=p_{\text {out }}, \quad \frac{\partial u}{\partial x}(L, y)=0, \quad \frac{\partial v}{\partial x}(L, y)=0, \quad \frac{\partial T}{\partial x}(L, y)=0
$$


The symmetry boundary conditions are considered on the channel axis at $y=0$ :

$$
\frac{\partial u}{\partial y}(x, 0)=0, \quad v(x, 0)=0, \quad \frac{\partial T}{\partial y}(x, 0)=0
$$

On the gas side of the impermeable wall at $y=H / 2$ (denoted by the subscript $g$ below), zero normal velocity is applied and, for the tangential velocity, the rarefaction effects are modeled using the first-order Maxwell slip velocity and thermal creep boundary conditions (Eq. (4)):

$$
\begin{gathered}
u_{g}(x)=u(x, H / 2)=\underbrace{-\left.\sigma_{\mu} \lambda_{g} \frac{\partial u}{\partial y}\right|_{g}}_{1^{\text {st }} \text { order slip velocity }}+\underbrace{\left.\sigma_{\theta} \frac{\mu_{g}}{\rho_{g} T_{g}} \frac{\partial T}{\partial x}\right|_{g}}_{\text {thermal creep }} \\
v_{g}(x)=v(x, H / 2)=0
\end{gathered}
$$

where the mean free path $\lambda_{g}$ at the wall is viscosity based (Eq. (1)):

$$
\lambda_{g}=\frac{\mu_{g}}{p_{g}} \sqrt{\frac{\pi r T_{g}}{2}}=\mu_{g} \sqrt{\frac{\pi}{2 \rho_{g} p_{g}}}
$$

For a channel with a uniform wall temperature $T_{w}$, the thermal boundary condition (6) is used:

$$
T_{g}(x)=T(x, H / 2)=T_{w}-\underbrace{\left.\xi_{T} \lambda_{g} \frac{\partial T}{\partial y}\right|_{g}}_{1^{\text {st }} \text { order temperature jump }}
$$

\subsection{Dimensionless equations for flows in isothermally-heated, long micro-channels}

\section{Reference quantities}

In the present study, we consider developed gas flows (i.e. located downstream the thermal entrance zone), in micro-channels isothermally heated at the wall temperature, $T_{w}$. Thus the following dimensionless variables are used in order to write the conservation equations and boundary conditions in dimensionless form :

$$
\begin{gathered}
X=x / L, Y=y / D_{h}, \quad U=u / u_{r e f}, V=v / u_{r e f}, \quad P=\frac{p-p_{a v}}{\Delta p_{r e f}}, \theta=\frac{T-T_{w}}{\Delta T_{r e f}} \\
\rho^{*}=\rho / \rho_{w}, \quad \mu^{*}=\mu / \mu_{w}, \quad C_{p}^{*}=C_{p} / C_{p, w} \quad, \quad k^{*}=k / k_{w}
\end{gathered}
$$

The subscript " $w$ " denotes quantities evaluated at the wall temperature, with $\rho_{w}=p_{a v} / r T_{w}$ and $p_{a v}=\left(p_{\text {in }}+p_{\text {out }}\right) / 2 ; u_{\text {ref }}, \Delta p_{\text {ref }}$ and $\Delta T_{\text {ref }}$ are reference quantities for the velocity, the pressure and temperature differences. In macro flows, the pressure gradient is generally considered of the same order as the inertial term, so the reference pressure difference is taken as $\Delta p_{\text {ref }}=\rho_{i n} \bar{u}_{\text {in }}^{2}$ for instance. In macro and micro channels, when the inlet and outlet pressures are the inputs of the problem formulation, the reference pressure is: 


$$
\Delta p_{\text {ref }}=p_{\text {in }}-p_{\text {out }}
$$

In dynamically developed flows in micro and macro channels, as the streamwise pressure gradient balances the viscous term, the equality of these two terms in the Navier-Stokes equations enables us to write the unknown reference velocity, $u_{r e f}$, as:

$$
u_{r e f}=\epsilon D_{h} \Delta p_{r e f} / \mu_{w}
$$

where $\epsilon=D_{h} / L=2 H / L$; it is a small parameter in the framework of the long channel approximation. Due to the way it is built, $u_{r e f}$ is a relevant reference velocity for the viscous term of the Navier-Stokes equations or for the viscous dissipation term of the energy equation.

On the other hand, the average or bulk velocity remains the adequate reference velocity for the inertial terms and the Reynolds and Mach numbers. Thus, in what follows, a bulk velocity, $\bar{u}_{b}$, defined from the mass flow rate, $\dot{m}$, will also be used:

$$
\bar{u}_{b}=\dot{m} / \rho_{w} H
$$

Note that $\dot{m}$ is a priori unknown for an imposed pressure difference, $p_{\text {in }}-p_{\text {out }}$. It can be estimated from the asymptotic analytical expression Eq. (63) for instance.

For a uniform wall temperature, $T_{w}$, the reference temperature difference is usually chosen as $\Delta T_{\text {ref }}=T_{w}-T_{i n}$. Here, we are mainly interested in the fully developed micro-flows downstream the thermal entrance length. Far from the inlet and for isothermal channel walls, the thermal scale can be chosen by assuming that the thermal diffusion balances the heat source terms (pressure work and viscous dissipation) in the energy equation (24) (such an assumption will be justified later on). Then we get:

$$
\Delta T_{r e f}=\frac{\mu_{w} u_{r e f}^{2}}{k_{w}}
$$

where $u_{r e f}$ is given by Eq. (35).

\section{Dimensionless equations and boundary conditions}

After introducing the dimensionless variables (33), taking into account Eqs. (34)-(37), the governing equations (21)-(24) become:

$$
\epsilon \frac{\partial \rho^{*} U}{\partial X}+\frac{\partial \rho^{*} V}{\partial Y}=0
$$




$$
\begin{gathered}
\epsilon \frac{\partial \rho^{*} U U}{\partial X}+\frac{\partial \rho^{*} U V}{\partial Y}=-\frac{1}{R e_{w}} \frac{\bar{u}_{b}}{u_{r e f}} \frac{\partial P}{\partial X}+\frac{1}{R e_{w}} \frac{\bar{u}_{b}}{u_{r e f}}\left\{\frac{\partial}{\partial X}\left[2 \mu^{*}\left(\epsilon^{2} \frac{\partial U}{\partial X}-\frac{1}{3}\left(\epsilon^{2} \frac{\partial U}{\partial X}+\epsilon \frac{\partial V}{\partial Y}\right)\right)\right]+\right. \\
\left.\frac{\partial}{\partial Y}\left[\mu^{*}\left(\frac{\partial U}{\partial Y}+\epsilon \frac{\partial V}{\partial X}\right)\right]\right\} \\
\epsilon \frac{\partial \rho^{*} U V}{\partial X}+\frac{\partial \rho^{*} V V}{\partial Y}=-\frac{1}{\epsilon R e_{w}} \frac{\bar{u}_{b}}{u_{r e f}} \frac{\partial P}{\partial Y}+\frac{1}{R e_{w}} \frac{\bar{u}_{b}}{u_{r e f}}\left\{\frac{\partial}{\partial X}\left[\mu^{*}\left(\epsilon \frac{\partial U}{\partial Y}+\epsilon^{2} \frac{\partial V}{\partial X}\right)\right]+\right. \\
\left.\frac{\partial}{\partial Y}\left[2 \mu^{*}\left(\frac{\partial V}{\partial Y}-\frac{1}{3}\left(\epsilon \frac{\partial U}{\partial X}+\frac{\partial V}{\partial Y}\right)\right)\right]\right\} \\
C_{p}^{*}\left(\epsilon \frac{\partial \rho^{*} U \theta}{\partial X}+\frac{\partial \rho^{*} V \theta}{\partial Y}\right)=\frac{1}{P r_{w} R e_{w}} \frac{\bar{u}_{b}}{u_{r e f}}\left[\epsilon^{2} \frac{\partial}{\partial X}\left(k^{*} \frac{\partial \theta}{\partial X}\right)+\frac{\partial}{\partial Y}\left(k^{*} \frac{\partial \theta}{\partial Y}\right)\right]+ \\
\frac{1}{P r_{w} R e_{w}} \frac{\bar{u}_{b}}{u_{r e f}}\left(U \frac{\partial P}{\partial X}+\frac{1}{\epsilon} V \frac{\partial P}{\partial Y}\right)+\frac{1}{P r_{w} R e_{w}} \frac{\bar{u}_{b}}{u_{r e f}} \Phi_{v}
\end{gathered}
$$

where $\Phi_{v}$ is the dimensionless viscous dissipation term (25):

$$
\Phi_{v}=\mu^{*}\left\{2\left[\left(\epsilon \frac{\partial U}{\partial X}\right)^{2}+\left(\frac{\partial V}{\partial Y}\right)^{2}\right]+\left[\frac{\partial U}{\partial Y}+\epsilon \frac{\partial V}{\partial X}\right]^{2}-\frac{2}{3}\left[\epsilon \frac{\partial U}{\partial X}+\frac{\partial V}{\partial Y}\right]^{2}\right\}
$$

In Eqs. (38)-(42), $\epsilon=D_{h} / L$ is a small parameter for most micro channels. $P r_{w}=\mu_{w} C_{p, w} / k_{w}$ and $R e_{w}=\rho_{w} \bar{u}_{b} D_{h} / \mu_{w}=2 \dot{m} / \mu_{w}$ are the Prandtl and Reynolds numbers evaluated at the wall temperature. So the last two terms of the energy equation (41), the pressure work $(P W)$ and viscous dissipation $(V D)$ terms, are both of the order of $\bar{u}_{b} /\left(\operatorname{Pr}_{w} R e_{w} u_{r e f}\right)$, considering that, from Eq. (38), $V \sim \epsilon$ in the $P W$ term. The ratio $P W / V D$ is therefore of the order of -1 since $P W$ is negative and $V D$ is positive.

The dimensionless form of the state equation is:

$$
\rho^{*}=\frac{1+\frac{\Delta p_{r e f}}{p_{a v}} P}{1+\frac{\Delta T_{r e f}}{T_{w}} \theta}
$$

The dimensionless forms of the boundary conditions read:

- on the inlet, at $X=0, \forall Y \in\left[0, \frac{1}{4}\right], P=P_{\text {in }}=\frac{1}{2}, \frac{\partial U}{\partial X}=V=0, \theta=\theta_{\text {in }}=\frac{T_{w}}{\Delta T_{\text {ref }}}\left(\frac{T_{i n}}{T_{w}}-1\right)$;

- on the outlet, at $X=1, \forall Y \in\left[0, \frac{1}{4}\right], P=P_{\text {out }}=-\frac{1}{2}, \frac{\partial U}{\partial X}=\frac{\partial V}{\partial X}=\frac{\partial \theta}{\partial X}=0$;

- on the channel axis, at $Y=0, \forall X \in[0,1], \frac{\partial U}{\partial Y}=V=\frac{\partial \theta}{\partial Y}=0$;

- on the wall, at $Y=\frac{1}{4}, \forall X \in[0,1]$, the velocity boundary conditions (29) and (30) write:

$$
U_{g}=\left.\frac{-\sigma_{\mu} K n_{w} \mu_{g}^{*}}{\sqrt{\rho_{g}^{*}\left(1+\frac{\Delta p_{r e f}}{p_{a v}} P_{g}\right)}} \frac{\partial U}{\partial Y}\right|_{g}+\left.\sigma_{\theta}\left(\frac{2(\gamma-1)}{\pi \gamma}\right) \epsilon P r_{w} K n_{w}^{2} R e_{w}\left(\frac{u_{r e f}}{\bar{u}_{b}}\right)\left(\frac{\mu_{g}^{*}}{1+\frac{\Delta p_{r e f}}{p_{a v}} P_{g}}\right) \frac{\partial \theta}{\partial X}\right|_{g}
$$

$$
V_{g}=0
$$


where all the variables with subscript $g$ are functions of $X$ at $Y=\frac{1}{4}$, on the gas side. Equation (44) shows that the thermal creep (the last R.H.S term) is $O(\epsilon)$ and $O\left(K n_{w}^{2}\right)$ that is of second order with $K n_{w}$ when the slip velocity (the first R.H.S term) is $O\left(K n_{w}\right)$. Finally, in the case of uniform wall temperature $T_{w}$, the thermal boundary condition (32) on the wall at $Y=\frac{1}{4}$ writes, $\forall X \in[0,1]$ :

$$
\theta_{g}=\left.\frac{-\xi_{T} K n_{w} \mu_{g}^{*}}{\sqrt{\rho_{g}^{*}\left(1+\frac{\Delta p_{r e f}}{p_{a v}} P_{g}\right)}} \frac{\partial \theta}{\partial Y}\right|_{g}
$$

and the resulting total wall heat flux, $q_{t, w}$, is given by Eq. (18) which also writes:

$$
q_{t, w}^{*}=\frac{q_{t, w} D_{h}}{k_{w} \Delta T_{r e f}}=\left.k_{g}^{*} \frac{\partial \theta}{\partial Y}\right|_{g}+\left.\mu_{g}^{*} U_{g} \frac{\partial U}{\partial Y}\right|_{g}
$$

\section{Number of independent parameters}

In the above dimensionless conservation equations and boundary conditions (Eqs. (38)-(46)), the following set of 12 dimensionless parameters emerges:

- $\operatorname{Pr}_{w}=\frac{\mu_{w} C_{p, w}}{k_{w}}$

- $R e_{w}=\frac{\rho_{w} \bar{u}_{b} D_{h}}{\mu_{w}}=\frac{2 \dot{m}}{\mu_{w}}$

- $K n_{w}=\frac{\lambda_{w}}{D_{h}}=\sqrt{\frac{\pi}{2}} \frac{\mu_{w}}{D_{h}} \frac{\sqrt{r T_{w}}}{p_{a v}}=\sqrt{\frac{\pi}{2}} \frac{\mu_{w}}{D_{h}} \frac{1}{\sqrt{\rho_{w} p_{a v}}}$

- $\frac{u_{r e f}}{\bar{u}_{b}}, \frac{\Delta p_{r e f}}{p_{a v}}, \frac{\Delta T_{r e f}}{T_{w}}, \frac{T_{w}}{T_{i n}}$

- $\epsilon=\frac{D_{h}}{L}, \sigma_{\mu}, \sigma_{\theta}, \xi_{T}$ and $\gamma$.

However, using the forthcoming analytical expression of the mass flow rate $\dot{m}$ (Eq. (63)), it can be checked that $u_{r e f}$ (Eq. (35)) is related to the reference average velocity $\bar{u}_{b}$ (Eq. (36)) by:

$$
\bar{u}_{b} / u_{r e f}=\frac{\left(1+12 \sigma_{\mu} K n_{w}\right)}{48}
$$

where $K n_{w}=\lambda_{w} / D_{h}$ is the Knudsen number, with the mean free path $\lambda_{w}$ evaluated from Eq. (1) at $T_{w}$ and at the average pressure $p_{a v}=\left(p_{\text {in }}+p_{\text {out }}\right) / 2$. Furthermore, by introducing the Mach number $M a_{w}^{2}=\rho_{w} \bar{u}_{b}^{2} / \gamma p_{a v}=\bar{u}_{b}^{2} / \gamma r T_{w}$, it can be shown that:

$$
\frac{1}{R e_{w}}=\sqrt{\frac{2}{\pi \gamma}} \frac{K n_{w}}{M a_{w}}
$$

$$
\frac{\Delta p_{r e f}}{p_{a v}}=\frac{\gamma}{\epsilon} \frac{M a_{w}^{2}}{R e_{w}} \frac{u_{r e f}}{\bar{u}_{b}} \quad \text { when } \Delta p_{r e f} \text { is given by Eqs. (34) }
$$

$$
\frac{\Delta T_{r e f}}{T_{w}}=(\gamma-1) \operatorname{Pr}_{w}\left(M a_{w} \frac{u_{r e f}}{\bar{u}_{b}}\right)^{2} \quad \text { when } \Delta T_{r e f} \text { is given by Eq. (37) }
$$


Therefore, if the micro channel is heated at constant temperature, the number of independent parameters is reduced to 9 . The following set of 9 parameters is used in what follows:

$$
P r_{w}, M a_{w}, K n_{w}, \frac{T_{w}}{T_{i n}}, \epsilon, \sigma_{\mu}, \sigma_{\theta}, \xi_{T}, \gamma
$$

Note that, if all the physical properties are assumed constant, the Reynolds number is constant in the whole channel due to mass flow rate conservation: $R e=R e_{w}=c s t e$. On the other hand, $M a$ and $K n$ vary along the flow in such a way that, from Eq. (49), their ratio is constant:

$$
\frac{M a}{K n}=\frac{M a_{w}}{K n_{w}}=\sqrt{\frac{2}{\pi \gamma}} R e=c s t e
$$

That means that when the compressibility effects increase ( $M a$ increases), the rarefaction effects decrease ( $K n$ decreases). Furthermore it is not possible to observe compressibility effects $(M a \sim$ $O(1))$ and rarefaction effects $\left(O\left(10^{-2}\right) \lesssim K n \lesssim O\left(10^{-1}\right)\right)$ at the same time when $\operatorname{Re} \lesssim O(1)$.

\section{Dimensional analysis and asymptotic solutions for long micro channels with isothermal walls}

A dimensional analysis of the mathematical model is useful to determine which terms are dominant or negligible. The case of developed, subsonic low-rarefied gas flows in isothermal long micro-channels of typical hydraulic diameter $D_{h} \sim 1$ to $10 \mu \mathrm{m}$ and length $L \sim 0.1$ to $10 \mathrm{~mm}$ at $\operatorname{Pr} \sim 1, M a<1$ and $10^{-3} \lesssim K n \lesssim 0.1$ is considered here. It is shown that, downstream the entrance zone, the viscous dissipation, pressure work and viscous stress power at the walls cannot be neglected in the mathematical model while the thermal creep is negligible (see Appendix A). Taking into account these results, we can compute an analytical solution of this flow type by solving a simplified model resulting from an asymptotic analysis. This analysis is valid for heated gaseous micro-flows, far from the thermal entrance effects, for long micro channels with isothermal walls. It shows that the Nusselt number tends to zero (and not to the usual value $N u_{\infty}=7.541$ ) for micro-flows between to flat plates. Here we only present and discuss the main results of this analysis for the present study (see Appendix B for more details).

Following the asymptotic analyzes by Arkilic et al. [62] and Cai et al. [63], considering weakly rarefied flows with small temperature variations in long micro-channels $\left(\epsilon=D_{h} / L<<1\right)$, the dimensionless quantities can be written as asymptotic expansions in $\epsilon$ :

$$
\begin{aligned}
& U=U_{0}+\epsilon U_{1}+\epsilon^{2} U_{2}+\ldots ; \quad V=\epsilon V_{1}+\epsilon^{2} V_{2}+\ldots ; \quad \theta=\epsilon \theta_{1}+\epsilon^{2} \theta_{2}+\ldots ; \\
& P=P_{0}+\epsilon P_{1}+\epsilon^{2} P_{2}+\ldots
\end{aligned}
$$

and the analytical solution of the asymptotic model for constant physical properties reads (see Appendix B):

$$
U_{0}(X, Y)=\frac{-1}{32} \frac{p_{a v}}{\Delta p_{r e f}} \frac{d P_{0}^{*}(X)}{d X}\left[1-16 Y^{2}+\frac{A}{P_{0}^{*}(X)}\right]=\frac{-1}{2} \frac{d P_{0}(X)}{d X}\left[b(X)-Y^{2}\right]
$$




$$
\begin{gathered}
V_{1}(X, Y)=\frac{A}{64} \frac{p_{a v}}{\Delta p_{\text {ref }}} \frac{1}{P_{0}^{*}(X)} \frac{d^{2} P_{0}^{*}(X)}{d X^{2}}\left[16 Y^{3}-Y\right]=\frac{A}{64} \frac{1}{P_{0}^{*}(X)} \frac{d^{2} P_{0}(X)}{d X^{2}}\left[16 Y^{3}-Y\right] \\
P_{0}^{*}(X)=1+\frac{\Delta p_{\text {ref }}}{p_{a v}} P_{0}(X)=\frac{1}{p_{a v}}\left\{\left(p_{\text {out }}^{2}-p_{\text {in }}^{2}\right)\left(1+\frac{3 A}{2}\right) X+\left(p_{\text {in }}+\frac{3 A}{2} p_{a v}\right)^{2}\right\}^{1 / 2}-\frac{3 A}{2} \\
\theta_{1}(X, Y)=-\frac{F(X)}{\epsilon}\left\{8 Y^{4}-16 b(X) Y^{2}+J(X)\right\}
\end{gathered}
$$

with

$$
\begin{gathered}
F(X)=\frac{1}{64}\left(\frac{d P_{0}(X)}{d X}\right)^{2} \\
b(X)=\frac{1}{16}\left[1+\frac{A}{P_{0}^{*}(X)}\right] \\
J(X)=\frac{1}{16} \frac{A}{P_{0}^{*}(X)}\left(1+\frac{B}{P_{0}^{*}(X)}\right)+\frac{1}{32} \\
A=8 \sigma_{\mu} K n_{w} \\
B=8 \xi_{T} K n_{w}
\end{gathered}
$$

Approximate analytical expressions of the mass flow rate, $\dot{m}$, and total wall Nusselt number, $N u_{t}(x)$, can also be deduced from this asymptotic analysis. At zero order with $\epsilon, \dot{m}$ reads as a function of $p_{\text {in }}$ and $p_{\text {out }}$ :

$$
\dot{m}=\frac{H^{3}}{24 L \mu_{w}} \frac{1}{r T_{w}}\left(p_{\text {in }}^{2}-p_{\text {out }}^{2}\right)\left[1+12 \sigma_{\mu} K n_{w}\right]=\rho_{w} u_{r e f} H \frac{1+12 \sigma_{\mu} K n_{w}}{48}
$$

For a constant wall temperature $T_{w}$, the total wall Nusselt number is defined by:

$$
N u_{t}(x)=N u_{\text {diff }}+N u_{V S P}=\frac{D_{h}}{k_{w}\left(T_{w}-\bar{T}(x)\right)}(\underbrace{-\left.k_{g} \frac{\partial T}{\partial \vec{n}}\right|_{g}}_{\text {diffusion heat flux }}-\underbrace{\left.\mu_{g} u_{g} \frac{\partial u}{\partial \vec{n}}\right|_{g}}_{\text {viscous stress power }})
$$

where the bulk temperatures is:

$$
\bar{T}(x)=\frac{2}{\dot{m}} \int_{0}^{H / 2} \rho(x, y) u(x, y) T(x, y) d y
$$

Thus $N u_{t}(x)$ is the sum of two contributions: the diffusive heat flux in the wall boundary layer, $N u_{d i f f}$, and the power of the viscous stress at the walls, $N u_{V S P}$. The asymptotic analysis in Appendix B shows that the viscous stress power at the wall is never equal to zero for $K n_{w} \neq 0$ and is the opposite of the conduction heat flux at the wall, at least at the first order with $\epsilon$, for $R e_{w}<O(1)$ and $M a_{w}<O(1)$. Consequently, under these conditions, $N u_{V S P}=-N u_{d i f f}$ and the total wall Nusselt number is zero:

$$
N u_{t}(x)=0
$$


It should be emphasized that these results are linked to the approximations used in deriving the analytical solution: the dynamical and thermal entrance effects are indeed neglected. In particular, the convective term and the streamwise variation of the temperature from inlet are not accounted for in the energy equation. This approximation is only justified in the case of long micro-channel because the Reynolds number is generally small and the thermal entrance length is thus very short (a few hydraulic diameters).

On the opposite, when convection is dominant while the pressure work and viscous dissipation are neglected in the energy equation, the Nusselt number can reach a finite value in a channel at constant wall temperature. For instance, for laminar incompressible flows between two infinite plates at constant wall temperature, it is well established in standard Heat Transfer textbooks that $N u(X \rightarrow \infty)=N u_{\infty}=7.541$ if $K n_{w}=0$ provided that the axial diffusion is neglected. Considering now both the viscous dissipation and the pressure work in the energy equation, our first order asymptotic analysis clearly shows that $N u(X)=0$ for $\operatorname{Re}_{w}<O(1)$ and $M a_{w}<O(1)$.

This theoretical analysis could then explain the very small values of the Nusselt numbers obtained by Demsis et al. $[23,24]$ in their experiments. The authors used a standard tubein-tube heat exchanger in which a rarefied cold gas flows in the interior tube and hot water in the exterior annular tube. The thermal conditions for the rarefied gas correspond to flows in a nearly isothermal pipe. The total wall heat transfer coefficient $h$ is determined through the measurements of the inlet and outlet gas and water temperatures of the tube-in-tube heat exchanger. For four rarefied gas flows (nitrogen, oxygen, argon and helium) in the range of Knudsen number $0.0022-0.032$ and range of Reynolds numbers $0.13-14.7$, the obtained Nusselt numbers vary between $6.2 \times 10^{-5}$ and $2.8 \times 10^{-2}$. This is two to five orders of magnitude smaller than the corresponding values in the continuum regime since in an isothermal duct the fully-developed Nusselt number at large Péclet numbers is $N u_{\infty}=3.66$.

Till now, these very small values of the Nusselt number had never been explained theoretically because only the contribution of the diffusion heat flux at the walls was considered while the contribution of the viscous stress power was always omitted in the wall total heat flux. In what follows, we are going to check the validity of the asymptotic solution, based on an approximate model, by numerically solving the full system of governing equations for compressible micro-flows in parallel plate micro-channels.

\section{Analysis of the heat transfer balance from numerical simulations}

\subsection{Numerical method.}

An in-house finite volume code has been developed to solve the steady Navier-Stokes and energy equations with first-order slip boundary conditions (Eqs. (21)-(32) in §3.5) on unstructured meshes. Details upon the discretization of the different terms can be found in [64-66]. Only a few points are reminded here. A second-order centered scheme is used for the diffusive and convective terms because the maximum Reynolds number is $R e_{\max }<15$ and cell Reynolds number is $R e=\frac{u \Delta x}{\nu}<1$; the same inequalities also apply for the Péclet number since 
$\operatorname{Pr} \leq \mathcal{O}(1)$. The discrete nonlinear steady equations are solved in a coupled way by Newton's algorithm. Since the channel geometry is here reduced to a rectangular domain, a Cartesian grid is used. The mesh size is based on the half channel width, with symmetry conditions (see Fig. 2). Mesh sizes are provided in each of the next subsections, according to the studied problem. For each case, a particular attention will be paid to the relevance of the results by checking the sensitivity of the solutions to the mesh refinement. The code has already been validated by numerous comparisons with experimental and numerical results [67-70].

\subsection{Comparison of the analytical and numerical solutions}

In this section, we compare the numerical and analytical solutions (Eqs. (54)-(62) in §4). The comparisons are carried out on three characteristics micro-flows, slipping or not, compressible or not. The number of control volume on each space direction is $N_{x} \times N_{y}=12000 \times 60$ for a 100-aspect ratio physical domain. The mesh is uniform on $x$-direction and refined near the wall on $y$-direction, with a size ratio between two successive cells equal to 0.975 .

\subsubsection{Studied cases}

The numerical simulations are performed for a nitrogen flow at $T_{i n}=270 \mathrm{~K}$ in a microchannel of length $L=300 \mu \mathrm{m}$ and height $H=3 \mu \mathrm{m}$, with walls at $T_{w}=300 \mathrm{~K}$. The physical properties of the gas are assumed constant and their values at $T_{w}$ are gathered in Table 1 . The three simulated cases are presented in Table 2:

- Case (a) is at low pressure and corresponds to a quasi incompressible flow $(M a<0.09)$ but with a strong slip velocity all along the channel walls because the Knudsen number values are relatively large, both at the inlet and outlet sections $(0.02<K n<0.1)$;

- Case (b) is at a large pressure difference between inlet and outlet. It corresponds to a strongly compressible flow since $M a_{\text {out }}=0.6428$ and it presents a strong slip at the channel outlet since $K n_{\text {out }}=0.09354$;

- Case (c) is at high pressure but small relative difference in the inlet and outlet pressures. It corresponds to an incompressible flow with a very low slip since $M a<0.03$ and $K n<$ 0.0023 all along the channel.

\begin{tabular}{|c|c|c|c|c|c|c|c|c|}
\hline $\begin{array}{c}k \\
{[W / m . K]}\end{array}$ & $\begin{array}{c}C_{p} \\
{[\mathrm{~J} / \mathrm{kg} \cdot \mathrm{K}]}\end{array}$ & $\begin{array}{c}\mu \times 10^{5} \\
{[\text { Pa.s }]}\end{array}$ & $\operatorname{Pr}$ & $\begin{array}{c}r \\
{[J / k g . K]}\end{array}$ & $\begin{array}{c}\gamma= \\
C_{p} / C_{v}\end{array}$ & $\sigma_{\mu}$ & $\sigma_{\theta}$ & $\xi_{T}$ \\
\hline 0.024712 & 1032.48 & 1.6588 & 0.693 & 296.8 & 1.4 & 1 & 1 & 2 \\
\hline
\end{tabular}

Tab. 1: Nitrogen physical properties at $T_{w}=300 \mathrm{~K}$.

\subsubsection{Comparisons of the velocity and temperature fields}

Table 3 gives the relative errors on the mass flow rate, $e_{\dot{m}}$, between the analytical solution of Eq. (89) and the numerical solutions in Table 2. An excellent agreement is observed since 


\begin{tabular}{|c|c|c|c|c|c|c|c|c|c|c|}
\hline Case & $\begin{array}{c}p_{\text {in }} \\
{[\text { bar }]}\end{array}$ & $\begin{array}{c}p_{\text {out }} \\
{[\text { bar }]}\end{array}$ & $\begin{array}{c}\Delta p_{\text {ref }} \\
{[\text { bar }]}\end{array}$ & $\begin{array}{c}R e_{\text {in }}= \\
R e_{\text {out }}\end{array}$ & $K n_{\text {in }}$ & $K n_{\text {out }}$ & $M a_{\text {in }}$ & $M a_{\text {out }}$ & $\begin{array}{c}\dot{m} \times 10^{6} \\
{[\mathrm{~kg} / \mathrm{m} . \mathrm{s}]}\end{array}$ & $\overline{N u_{t}}$ \\
\hline (a) & 0.5 & 0.1 & 0.4 & 1.038 & 0.019620 & 0.1032 & 0.01373 & 0.08848 & 8.608 & 0.1082 \\
(b) & 1.5 & 0.1 & 1.4 & 7.833 & 0.006539 & 0.09354 & 0.03453 & 0.64280 & 64.91 & 0.1472 \\
(c) & 5 & 4.6 & 0.4 & 12.05 & 0.001962 & 0.002248 & 0.01595 & 0.02716 & 99.97 & 0.2215 \\
\hline
\end{tabular}

Tab. 2: Imposed inlet and outlet pressures for each simulated case. The others quantities, except $K n_{i n}$, result from the simulations.

the maximum relative error is $1.2 \%$ for the case $(b)$.

\begin{tabular}{|c|c|c|c|c|c|c|}
\hline Case & $p_{\text {in }}[$ bar $]$ & $p_{\text {out }}[$ bar $]$ & $e_{\dot{m}}[\%]$ & $e_{u}[\%]$ & $e_{p}[\%]$ & $e_{T}[\%]$ \\
\hline (a) & 0.5 & 0.1 & 0.06 & 0.32 & 0.040 & 0.43 \\
(b) & 1.5 & 0.1 & 1.2 & 6.9 & 0.68 & 0.53 \\
(c) & 5 & 4.6 & 0.04 & 0.68 & $4.6 \times 10^{-4}$ & 0.54 \\
\hline
\end{tabular}

Tab. 3: Relative error on $\dot{m}$ and $L_{2}$-norm of the relative errors on $u, p$, and $T$ between the analytical and numerical solutions.

The comparison of the analytical and numerical solutions for the streamwise, $u$, and transverse, $v$, velocity components and the temperature, $T$, are presented in Figures 3-5 for the cases (a)-(c) respectively. Table 3 also gives the $L_{2}$-norm of the relative errors on $u, p$, and $T$ between the analytical and numerical solutions. The values and absolute errors on $v$ being both very small, the relative errors on $v$ are not significant and then not presented here. Globally, the analytical and numerical solutions for $u, p$ and $T$ are in a good accordance since the maximum relative error is $6.9 \%$ on $u$ in the case (b). The graphical comparisons show a good agreement between the solutions, except at the outlet in the compressible case (b) (Fig. 4) and at the inlet for the temperature field. In the former case, the Reynolds and Mach numbers are relatively large $\left(R e=7.8\right.$ and $\left.M a_{\text {out }}=0.64\right)$ : the upper limit of the validity domain of the asymptotic analysis is reached $\left(R e_{w} \lesssim O(1), M a_{w}<O(1)\right)$. In the later case, the inlet and wall temperatures differ from each other in the numerical simulations, what produces a thermal entrance length which is not considered in the used analytical model. To a lesser extent, the entrance region also affects the $v$-field in Fig 5 but, in this case, the $v$-value remains very small.

\subsubsection{Comparisons of the Nusselt number values}

The wall total Nusselt number, $N u_{t}(x)=N u_{\text {diff }}(x)+N u_{V S P}(x)$, is defined by Eq. (64). The integral average of $N u_{t}(x)$ along the wall is denoted by $\overline{N u_{t}}$ and the numerical values for the cases (a) to (c) are reported in Table 2. They are very low $\left(\overline{N u_{t}} \sim 0.1\right)$ compared to the standard value $N u_{\infty}=7.541$ calculated for a fully-established incompressible flow at large Péclet numbers between two isothermal parallel plates. The computed $\overline{N u_{t}}$-values are in qualitative agreement with the zero value of the total Nusselt number predicted analytically (Eq. (99)), by neglecting the thermal entrance length and keeping the leading terms of the $\epsilon$-expansion. Furthermore, by comparing the cases (c) and (a) in Table 2, it appears that $\overline{N u_{t}}$ 


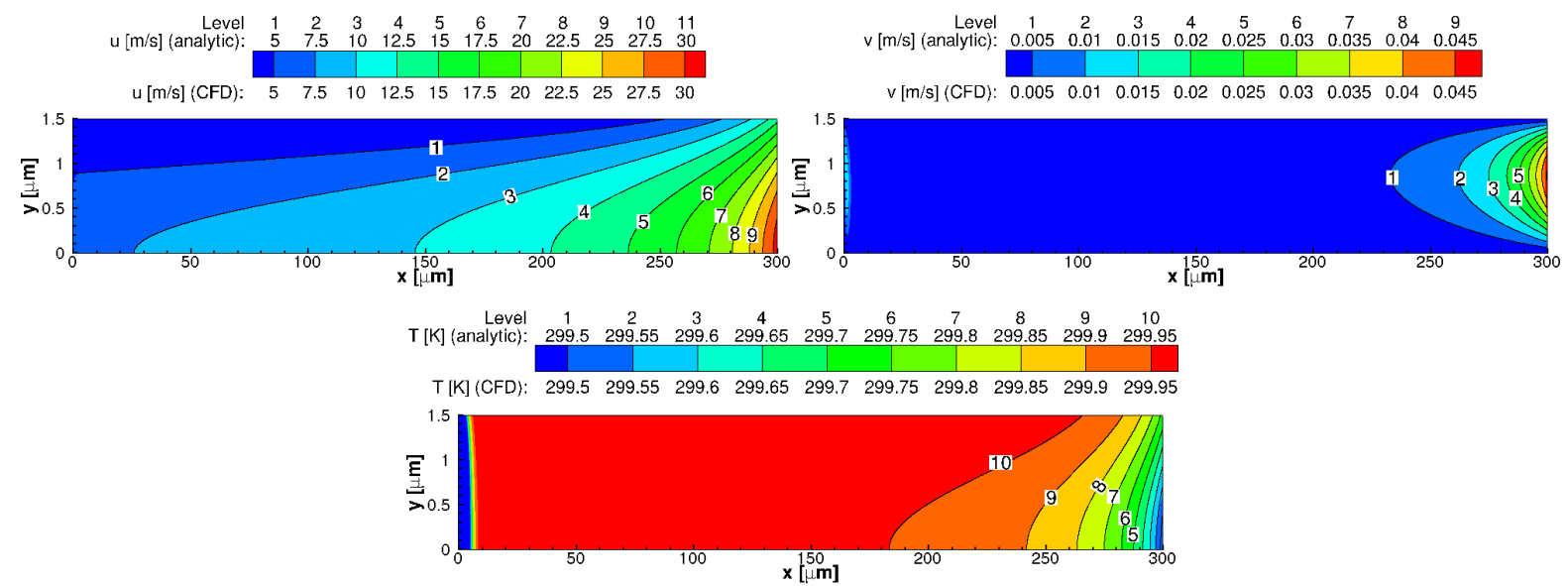

Fig. 3: Comparison of the analytical solution (black isolines) and numerical solution (color fields) for $u$ (top, left), $v$ (top, right) and $T$ (bottom), in case (a). Symmetry is taken into account and a half channel is presented.

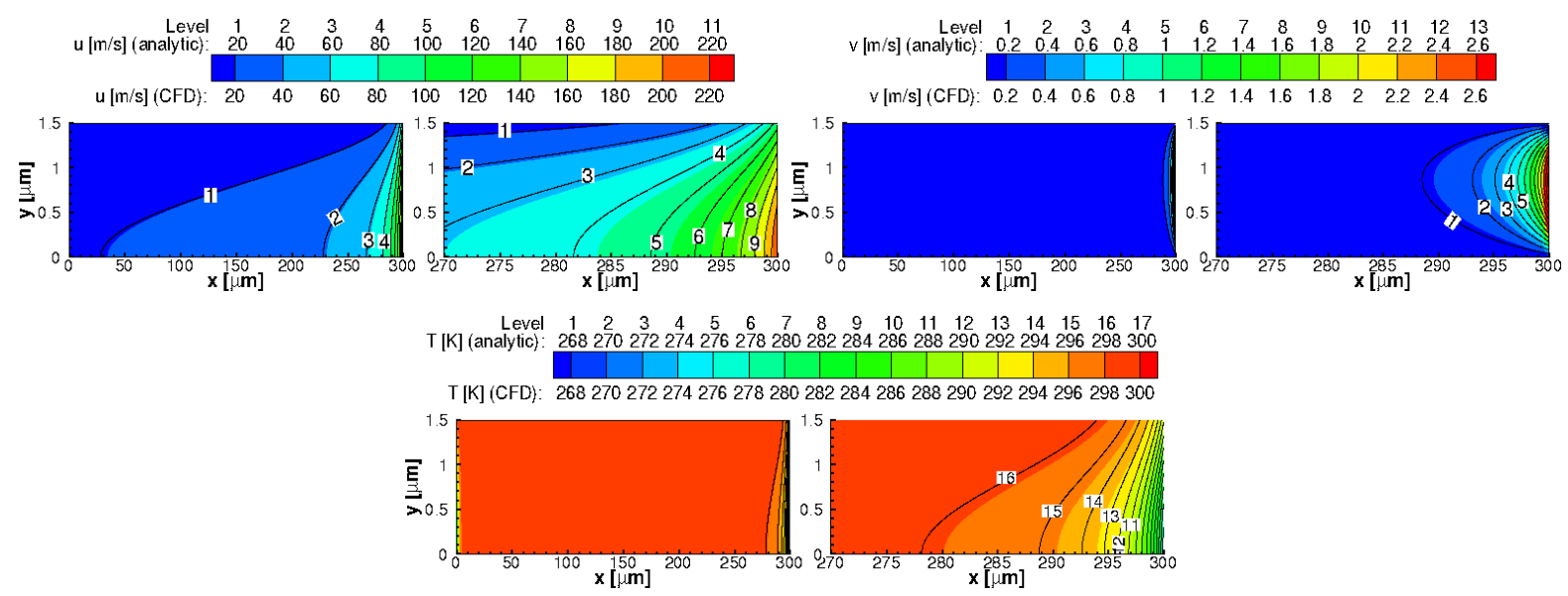

Fig. 4: Same as Figure 3 but for case (b) and with a zoom on the outlet zone for each field (see coordinates on the abscissa axes).

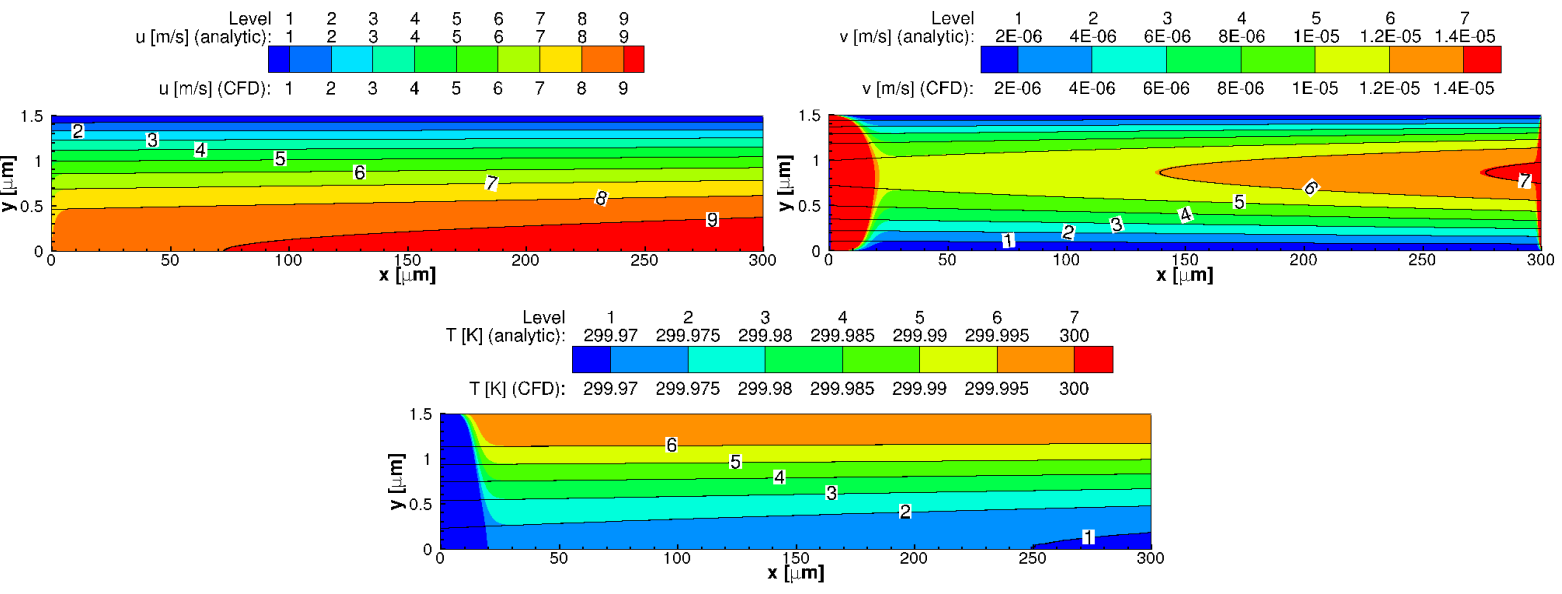

Fig. 5: Same as Figure 3 but for case (c).

decreases when $K n$ increases.

The profiles of $N u_{t}, N u_{d i f f}$ and $N u_{V S P}$, computed from the numerical simulations, are 
plotted with respect to $x / L$ on Figs. 6-8 for the cases (a)-(c), respectively. From these profiles and from Figs. 3-5, it appears that the length of the thermal entrance is approximately equal to $5 \mathrm{H}$ in the low Reynolds number case (a) (Fig. 6) and to $10 \mathrm{H}$ at maximum in the higher Reynolds number case (c) (Fig. 8). The total local Nusselt number, $N u_{t}$, is very small in the whole channel except at the entrance where the flow is not thermally-established, and close to the outlet section due to the gas expansion and cooling associated with the pressure work. Downstream the thermal entrance zone, $N u_{\text {diff }}$ and $-N u_{V S P}$ vary between $\sim 0.2$ in case (c) (Fig. 8) and $\sim 1.5$ in case (a) (Fig. 6), while $N u$ varies between less than 0.002 in case (a) and less than 0.03 in case (b) (Fig. 7). Thus even though $N u_{d i f f}$ and $N u_{V S P}$ take non negligible values, $N u_{t}=N u_{\text {diff }}+N u_{V S P}$ nearly vanishes because $N u_{V S P}$ is nearly the opposite of $N u_{\text {diff }}$ downstream the thermal entrance zone (note that it is " $-N u_{\text {diff }}$ " that is plotted on Figs. 6-8). These Nusselt number behaviors are investigated in more details in the next section.

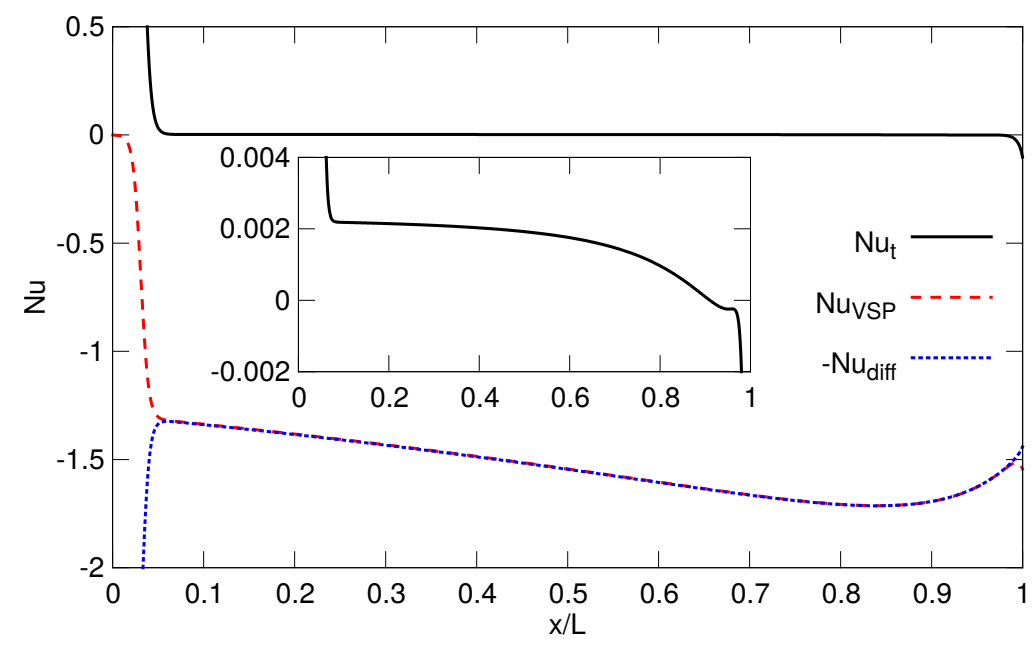

Fig. 6: Profiles of $N u_{t}, N u_{V S P}$ and $-N u_{d i f f}$, with respect to $x / L$, in the simulated case (a). A zoom on the $N u_{t}$ profile is included.

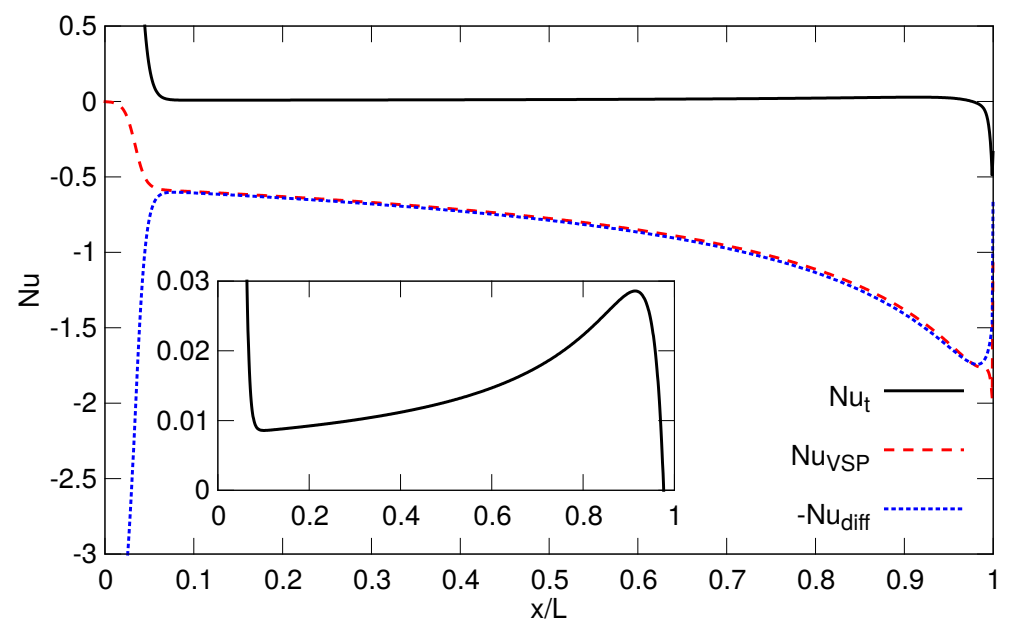

Fig. 7: Same as Figure 6 but for case (b). 


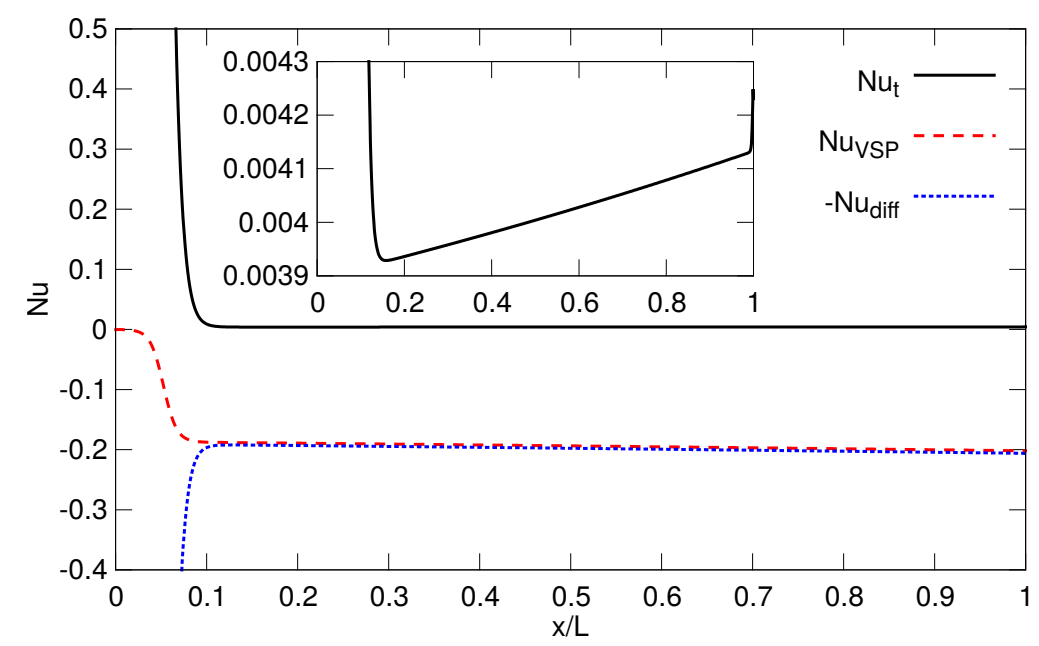

Fig. 8: Same as Figure 6 but for case (c).

\subsection{Detailed analysis of the Nusselt number}

The aim of this section is to understand why and how $N u_{t}$ tends to very small values in the preceding cases (a)-(c). We first investigate the classical case of an incompressible no slip flow between two infinite plates for which the standard Heat Transfer textbooks give $N u_{\infty}=7.541$.

\subsubsection{Nusselt number for incompressible no slip flows}

The numerical simulations are for a nitrogen flow at $T_{i n}=270 \mathrm{~K}$ and $T_{w}=300 \mathrm{~K}$ (see Table 1). The full numerical model is solved again. The pressure work $(P W)$ and viscous dissipation $(V D)$ source terms are kept in the energy equation. However, the Knudsen number is fixed here at $K n=0$ (no slip) and the density is fixed at $\rho_{a v}=\frac{p_{a v}}{r T_{w}}$, where $p_{a v}=\frac{p_{\text {in }}+p_{\text {out }}}{2}$ (incompressible flow). The pressure difference between the inlet and the outlet is very small $\left(\Delta p_{\text {ref }}=0.01\right.$ bar $)$ while the average pressure is high $\left(p_{a v}=4.995\right.$ bar $)$. Furthermore the channel aspect ratio is set at $L / H=50$ and the channel height varies between $H=3 \mu \mathrm{m}$ and $H=96 \mu \mathrm{m}\left(6 \mu \mathrm{m} \leq D_{h} \leq 192 \mu \mathrm{m}\right)$. The size of the uniform mesh is $N_{x} \times N_{y}=3000 \times 60$, except when it is stated that $N_{x} \times N_{y}=12000 \times 240$.

The Nusselt number and bulk temperature of incompressible no slip flows are defined by:

$$
\begin{gathered}
N u(x)=\frac{-\left.k \frac{\partial T}{\partial y}\right|_{w}}{k\left(T_{w}-\bar{T}(x)\right) / D_{h}} \\
\bar{T}(x)=\frac{2}{\bar{u} H} \int_{0}^{H / 2} u(x, y) T(x, y) d y
\end{gathered}
$$

The $N u$ profiles are plotted with respect to $x / L$ on Fig. 9, for various hydraulic diameters or Reynolds numbers. The Reynolds number, $R e_{D_{h}}$, is proportional to $D_{h}^{2}$ because the analytical average velocity, $\bar{u}$, is proportional to $D_{h}$ in simulations at fixed $\Delta p_{\text {ref }}$ and $L / H$ values. One can note that, when $D_{h}$ and $R e_{D_{h}}$ are large enough $\left(D_{h}=192 \mu \mathrm{m}\right.$ and $\left.R e_{D_{h}}=697\right), \mathrm{Nu}$ decreases at the channel entrance and tends towards the classical $N u$ value, $N u_{\infty}=7.541$, 
downstream. However, when $D_{h}$ (or $R e_{D_{h}}$ ) decreases, a different behavior is observed. The finite $N u$-value, reached after its sudden decrease close to the inlet section, tends towards zero further downstream. It is then possible to distinguish two entrance lengths: the first one is defined from the inlet section to the first Nusselt plateau and the second one to the fully established region where $N u \sim 0$. The length of the first plateau decreases with $D_{h}$ (or $R e_{D_{h}}$ ) as well as the length from the inlet to $N u \sim 0$. The explanation of $N u \sim 0$ is detailed below.

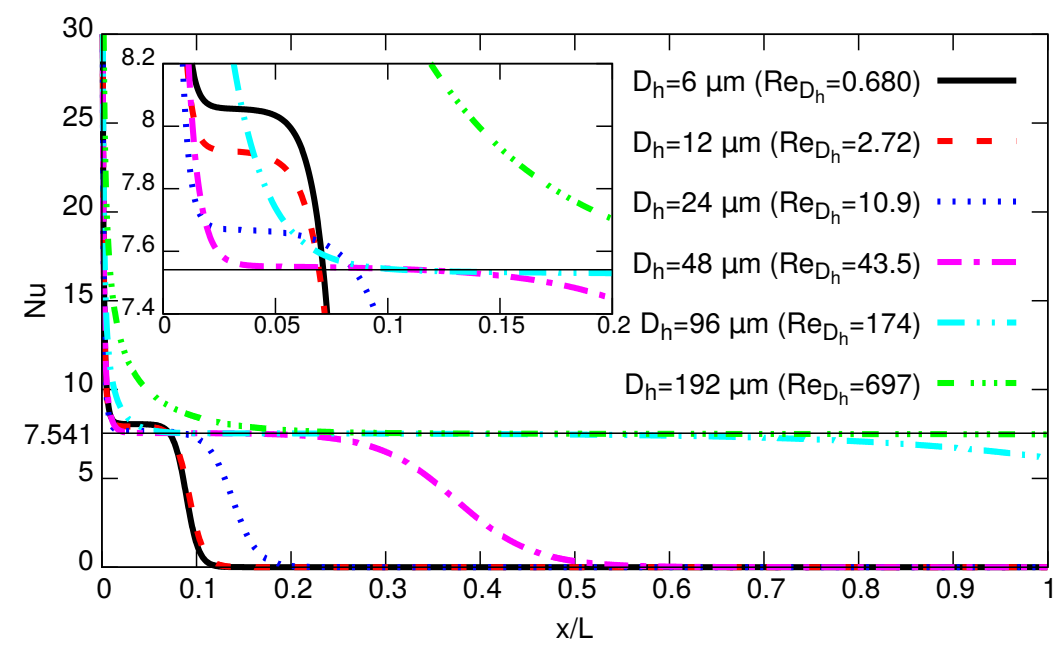

Fig. 9: Profiles of the wall Nusselt number, $N u$, with respect to $X=x / L$, for incompressible no slip flows at various hydraulic diameters and Reynolds numbers $\left(p_{\text {in }}=5 \mathrm{bar}, p_{\text {out }}=\right.$ $4.99 \mathrm{bar}, L / H=50)$. A zoom on the $N u$ profiles at the channel entrance is included.

Let us first reformulate the enthalpy equation (13) in its integral form as follows:

$$
\underbrace{\iint_{\delta \Sigma(x)}-\rho C_{p} T \vec{v} \cdot \vec{n} d S}_{=C O N V \times \Delta x}+\underbrace{\iint_{\delta \Sigma(x)} k \nabla T \cdot \vec{n} d S}_{=D I F F \times \Delta x}=\underbrace{\iiint_{\delta \Omega(x)} \vec{v} \cdot \nabla p d \omega}_{=P W \times \Delta x}+\underbrace{\iiint_{\delta \Omega(x)} \overline{\bar{\tau}}: \nabla \vec{v} d \omega}_{=V D \times \Delta x}
$$

where $\vec{n}$ is the inward unit vector, $\delta \Omega(x)$ is a channel slice of width $\Delta x$ at the mean abscissa $x$, and $\delta \Sigma(x)=S_{-}(x) \cup S_{+}(x) \cup \delta S_{w, g}(x) \cup \delta S_{s}(x)$ is its surface (see Fig. 2). The notations $C O N V$, $D I F F, P W$ and $V D$ refer to the average values per unit length $\Delta x$ of the CONVective and DIFFusive heat fluxes transferred through the surface $\delta \Sigma(x)$, and the average Pressure Work and Viscous Dissipation produced into the slice volume $\delta \Omega(x)$, respectively. The streamwise variations of these terms are plotted on Fig. 10 in the case of the narrowest channel of Fig. $9,\left(D_{h}=6 \mu \mathrm{m}\right)$. As the incompressible flow is dynamically fully-established from the inlet to the outlet of the channel, Fig. 10 shows that the $P W$ and $V D$ source terms are constant and strictly opposite all along the channel length: $P W(x)+V D(x)=0^{2}$. Since the flow tends to be thermally fully established far from the channel entrance $\left(\frac{\partial T}{\partial x} \rightarrow 0\right.$ and $\left.\frac{\partial \bar{T}}{\partial x} \rightarrow 0\right)$, the average convective term $C O N V$ also tends to zero, as well as the average diffusion heat flux $D I F F=P W+V D-C O N V \rightarrow 0$. Considering both this later equality, the streamwise diffusion

\footnotetext{
${ }^{2}$ This result has already been obtained and discussed at $\S 3.4$ where the L.H.S. term of Eq. (20) is equal to zero for dynamically fully-developed and incompressible no slip flows.
} 
heat flux which tends to zero, and the symmetry condition $\left(\left.\frac{\partial T}{\partial y}\right|_{s}=0\right)$, the diffusion heat flux at the wall necessarily tends towards zero too.

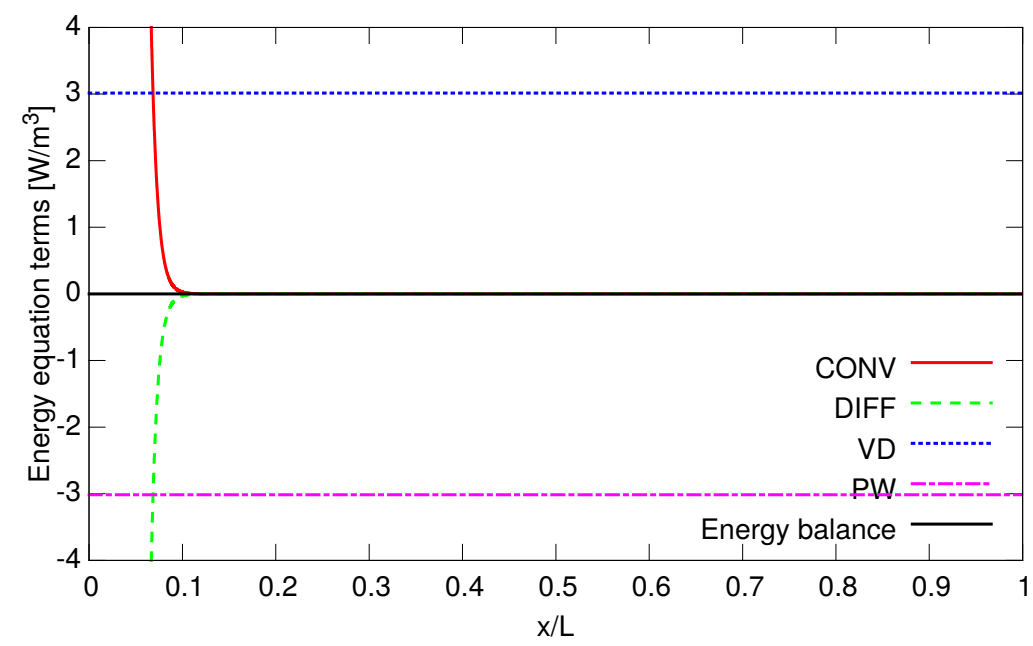

Fig. 10: Streamwise variation of each term of the energy equation integrated on a channel slice (convective $(C O N V)$ and diffusive $(D I F F)$ fluxes and pressure work $(P W)$ and viscous dissipation $(V D)$ source terms), and total energy balance $(C O N V+D I F F-P W-V D)$, for the no slip incompressible flow at $D_{h}=6 \mu \mathrm{m}, p_{\text {in }}=5 \mathrm{bar}, p_{\text {out }}=4.99 \mathrm{bar}$ of Fig. 9 .

This result is illustrated on Fig. 11 where both the numerator, $-\left.k \frac{\partial T}{\partial y}\right|_{w}$, and the denominator, $k\left(T_{w}-\bar{T}\right) / D_{h}$, of the Nusselt number expression (Eq. (67)) are plotted. It is worth noticing that the diffusion heat flux at the wall tends to zero with the grid refinement: past $x / L \sim 0.15$, its value is reduced by $4^{2}$ by dividing the Cartesian cell sizes by 4 , from $N_{x} \times N_{y}=3000 \times 60$ to $12000 \times 240$. The factor is equal to $4^{2}$ because the mesh size ratio is equal to four in each space direction and the space convergence order of the code is $O\left(\Delta x^{2}, \Delta y^{2}\right)$. This is highlighted by the black arrow between the red dashed line and the red square symbols. On the other hand, the Nusselt denominator, $k\left(T_{w}-\bar{T}\right) / D_{h}$, is insensitive to the mesh size and keeps a non-zero constant value for $x / L \gtrsim 0.15$. This temperature difference is related to the transverse thermal gradient induced by the source terms $P W$ and $V D$ in the integral energy equation (69). Indeed, though $P W$ and $V D$ counterbalance each other (Fig. 10), they contribute to the local thermal unbalance, acting close to the wall for the viscous dissipation and near the symmetry plane for the pressure work. Thus, as soon as the source terms dominate the convective and diffusive contributions for $x / L \gtrsim 0.75$ (Fig. 10), a constant transverse thermal gradient exists in the core flow. Consequently, the reference heat flux $k\left(T_{w}-\bar{T}\right) / D_{h}$ becomes constant and the Nusselt number cancels out (see the encapsulated graphic in Fig. 11).

\subsubsection{Nusselt number for incompressible slipping flows}

For an incompressible slipping flow, the Nusselt number behavior is similar to that of the incompressible no slip flow. This is illustrated by simulating a flow in a micro-channel at $D_{h}=6 \mu \mathrm{m}$, using the same conditions as in the previous subsection, except the Knudsen number that is computed by $K n=\lambda / D_{h}$ with $\lambda=\frac{\mu}{p} \sqrt{\frac{\pi r T}{2}}$, and the pressures that are equal to 


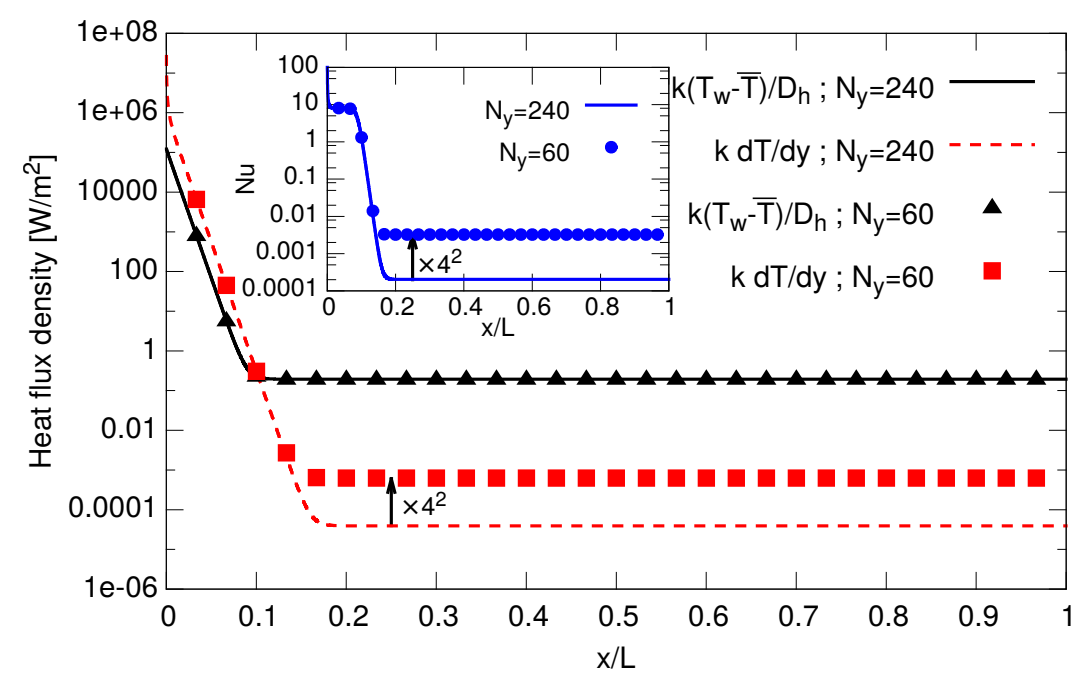

Fig. 11: Streamwise profiles of the Nusselt number $N u$ (enclosed figure), $N u$ numerator $\left(-\left.k \frac{\partial T}{\partial y}\right|_{w}\right)$ and $N u$ denominator $\left(k\left(T_{w}-\bar{T}\right) / D_{h}\right)$, for the incompressible no slip flow at $D_{h}=6 \mu \mathrm{m}, p_{\text {in }}=5 \mathrm{bar}$ and $p_{\text {out }}=4.99 \mathrm{bar}$ (Fig. 9) and for two uniform Cartesian meshes of sizes $N_{x} \times N_{y}=3000 \times 60$ and $12000 \times 240$.

$p_{\text {in }}=0.1$ bar and $p_{\text {out }}=0.09$ bar. The computed flow parameters are gathered in Table 4.

\begin{tabular}{|c|c|c|c|c|c|c|c|}
\hline $\begin{array}{c}p_{\text {in }} \\
{[\text { bar }]}\end{array}$ & $\begin{array}{c}p_{\text {out }} \\
{[\text { bar }]}\end{array}$ & $\begin{array}{c}\Delta p_{\text {ref }} \\
{[\text { bar }]}\end{array}$ & $\begin{array}{c}R e_{\text {in }}= \\
R e_{\text {out }}\end{array}$ & $K n_{\text {in }}$ & $K n_{\text {out }}$ & $\begin{array}{c}\dot{m} \times 10^{6} \\
{[\mathrm{~kg} / \mathrm{m} . \mathrm{s}]}\end{array}$ & $\overline{N u_{t}}$ \\
\hline 0.1 & 0.09 & 0.01 & 0.02823 & 0.1034 & 0.1088 & 0.2341 & 0.2039 \\
\hline
\end{tabular}

Tab. 4: Imposed inlet conditions and computed outlet conditions for the incompressible slipping flow in a micro-channel at $D_{h}=6 \mu \mathrm{m}$.

For a dynamically fully-developed incompressible flow, the integral of the total energy equation on the same channel slice $\delta \Omega(x)$ as in the previous subsection, of surface $\delta \Sigma(x)$ at the mean abscissa $x$ (see Fig. 2), writes (see Eq. (11)):

$$
\underbrace{\iint_{\delta \Sigma(x)}-\rho C_{p} T \vec{v} \cdot \vec{n} d S}_{=C O N V \times \Delta x}+\underbrace{\iint_{\delta \Sigma(x)} k \nabla T \cdot \vec{n} d S}_{=D I F F \times \Delta x}=\underbrace{\iint_{\delta \Sigma(x)}-(\overline{\bar{\tau}} \cdot \vec{v}) \cdot \vec{n} d S}_{=V S P \times \Delta x}
$$

where the $V S P$ term is the average power of the viscous stress on the slice surface $\delta \Sigma(x)$ per unit length. Assuming a fully-developed flow, this last term writes:

$$
V S P=\frac{1}{\Delta x} \iint_{\delta S_{w, g}(x)}-(\overline{\bar{\tau}} \cdot \vec{v}) \cdot \vec{n} d S=-(\overline{\bar{\tau}} \cdot \vec{v})_{y=H / 2} \cdot \vec{n}=\left.\mu_{g} u_{g} \frac{\partial u}{\partial y}\right|_{g}
$$

As the thermal energy balance (Eq. (69)) is still valid for an incompressible slipping flow, the thermal and mechanical energy balances write in a compact form:

$$
C O N V+D I F F=P W+V D=V S P
$$


The five terms of these equations are plotted on Fig. 12. It can effectively be checked that $P W+V D=C O N V+D I F F=V S P \neq 0$. When the flow tends to be thermally fullyestablished, that is when $\frac{\partial T}{\partial x} \rightarrow 0$, the convective term tends towards zero and the integral of the diffusive heat flux density tends towards the integral of the power of the viscous term at the wall: if $C O N V \rightarrow 0$ then $D I F F \rightarrow V S P \neq 0$. From a numerical point of view, the zoom in Fig. 12 shows that $D I F F \rightarrow V S P$ with a second order space convergence.

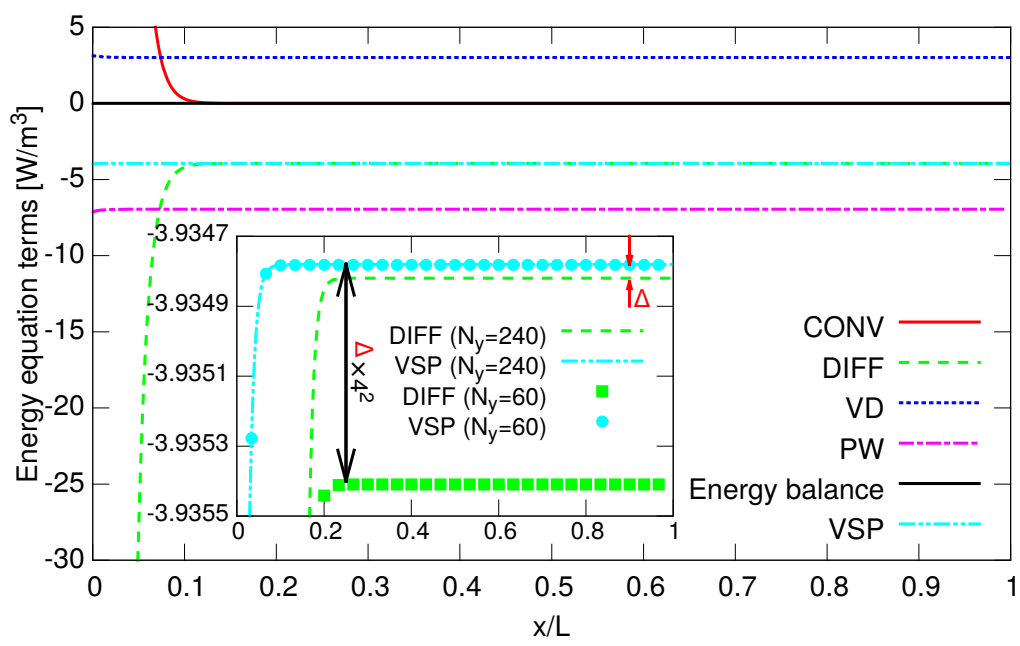

Fig. 12: Streamwise profiles of each term of Eq. (72) and energy balance $(C O N V+D I F F-$ $P W-V D)$, for the incompressible slipping flow at $D_{h}=6 \mu \mathrm{m}, p_{i n}=0.1 \mathrm{bar}$ and $p_{\text {out }}=0.09$ bar (see Table 4 ). The zoom shows that DIFF $\rightarrow V S P$ for two uniform Cartesian meshes of sizes $N_{x} \times N_{y}=3000 \times 60$ and $12000 \times 240$.

As a result, the total wall heat flux must tend to zero when the flow tends to be thermally fully-established because it is the sum of two opposite contributions at the walls: the diffusive flux and the power of the viscous stress (see Eq. (18)). Then the total local Nusselt number defined by $N u_{t}(x)=N u_{\text {diff }}(x)+N u_{V S P}(x)$ (Eq. (64)) must also tends towards zero. Indeed, as in the no slip case, the difference $\left(T_{w}-\bar{T}\right)$ at the $N u_{t}$ denominator still tends towards a non zero constant value (the $P W$ and $V D$ source terms do not cancel out and do not compensate each other locally). This is confirmed in Fig. 13 which presents the streamwise profiles of $N u_{t}$, $N u_{\text {diff }}$ and $N u_{V S P}$ for the incompressible slipping flow at $D_{h}=6 \mu \mathrm{m}$ (see Table 4), for two uniform Cartesian meshes of different sizes. $N u_{\text {diff }}$ and $N u_{V S P}$ tend towards opposite values after the thermal entrance zone and $N u_{t}$ tends towards zero. Once again, the second-order space convergence to a zero value of the total Nusselt number, $N u_{t}$, is checked on Fig. 13.

\subsubsection{Nusselt number for compressible slipping flows}

We now consider compressible slipping flows of nitrogen in a micro-channel of aspect ratio $L / H=50$, with $H=3 \mu \mathrm{m}\left(D_{h}=6 \mu \mathrm{m}\right)$. Not any simplification in the full model of $\S 3.5$ is invoked. The inlet and wall temperatures are $T_{i n}=270 \mathrm{~K}$ and $T_{w}=300 \mathrm{~K}$. The physical properties are given in Table 1 and the flow parameters in Table 5. The pressure difference 


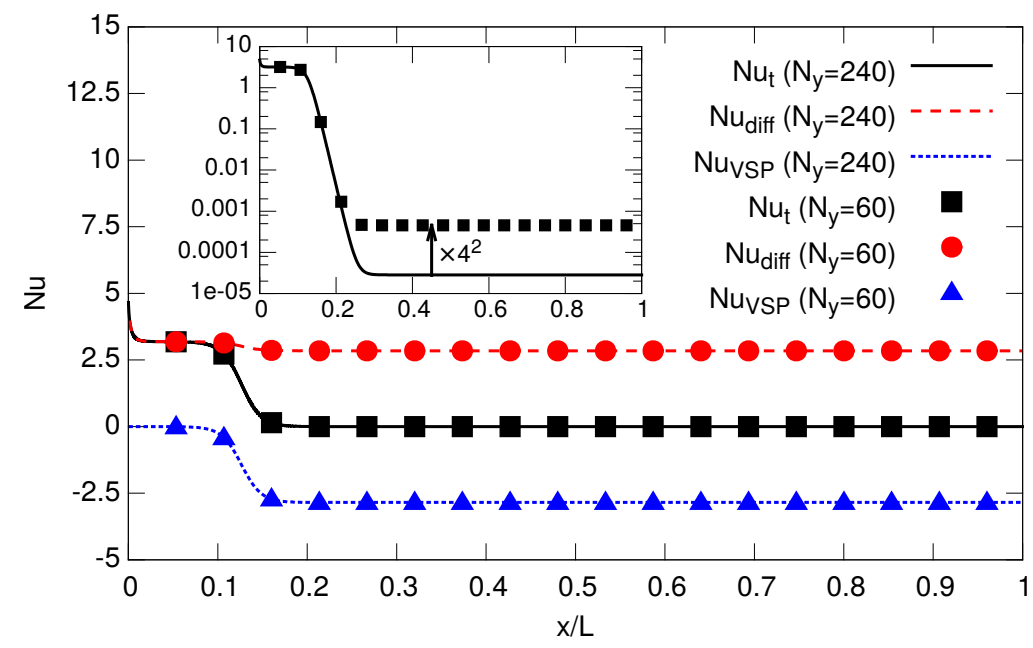

Fig. 13: Streamwise profiles of the total Nusselt number $N u_{t}(x)$, its diffusive part, $N u_{\text {diff }}(x)$, and its part due to $V S P, N u_{V S P}(x)$, for the incompressible slipping flow at $D_{h}=6 \mu \mathrm{m}$, $p_{\text {in }}=0.1 \mathrm{bar}$ and $p_{\text {out }}=0.09 \mathrm{bar}$ (see Table 4) and for two uniform Cartesian meshes of size $N_{x} \times N_{y}=3000 \times 60$ and $12000 \times 240$. A zoom on the $N u_{t}$ profiles is included.

between the inlet and the outlet is fixed at $\Delta p_{\text {ref }}=0.1$ bar but the average pressure decreases from one case to another one and, therefore, the average Knudsen number increases. The size of the uniform mesh is $N_{x} \times N_{y}=12000 \times 240$. It was checked to be enough fine to provide accurate solutions.

\begin{tabular}{|c|c|c|c|c|c|c|c|c|}
\hline $\begin{array}{c}p_{\text {in }} \\
\text { bar }]\end{array}$ & $\begin{array}{c}p_{\text {out }} \\
{[\text { bar }]}\end{array}$ & $\begin{array}{c}R e_{\text {in }}= \\
R e_{\text {out }}\end{array}$ & $K n_{\text {in }}$ & $K n_{\text {out }}$ & $M a_{\text {in }}$ & $M a_{\text {out }}$ & $\begin{array}{c}\dot{m} \times 10^{6} \\
{[\mathrm{~kg} / \mathrm{m} . \mathrm{s}]}\end{array}$ & $\overline{N u_{t}}$ \\
\hline 2 & 1.9 & 2.5402 & 0.00490 & 0.00544 & 0.01247 & 0.01370 & 21.07 & 0.2932 \\
1 & 0.9 & 1.3156 & 0.00981 & 0.01149 & 0.01268 & 0.01467 & 10.91 & 0.2598 \\
0.6 & 0.5 & 0.8257 & 0.01635 & 0.02068 & 0.01299 & 0.01613 & 6.848 & 0.2384 \\
0.3 & 0.2 & 0.4582 & 0.03270 & 0.05169 & 0.01380 & 0.02090 & 3.800 & 0.2048 \\
0.2 & 0.1 & 0.3357 & 0.04904 & 0.10336 & 0.01466 & 0.02864 & 2.785 & 0.1804 \\
0.15 & 0.05 & 0.2745 & 0.06539 & 0.20689 & 0.01555 & 0.04381 & 2.277 & 0.1611 \\
\hline
\end{tabular}

Tab. 5: Imposed inlet conditions and computed outlet conditions of the compressible flows simulated with the full model in a micro-channel at $D_{h}=6 \mu \mathrm{m}$ and $\Delta p_{\text {ref }}=0.1 \mathrm{bar}$.

An example of the energy balance is presented in Fig. 14 for the micro-flow at $p_{\text {in }}=0.2 \mathrm{bar}$ and $p_{\text {out }}=0.1$ bar $\left(5^{\text {th }}\right.$ row of Table 5 ). Here the flow is nearly incompressible since the maximum Mach number is $M a_{\text {out }} \approx 0.03$. However, the full compressible model is used for the simulation and weak compressible effects are highlighted in the energy balance. In the case of compressible micro-flows, the thermal energy balance $C O N V+D I F F=P W+V D$ is still valid but $P W+V D \neq V S P$. The simplified mechanical energy balance for incompressible flows (Eqs. (70) and (72)) is not valid because, due to the streamwise density variation, the velocity profile is not the same between two different channel sections. As a result, the kinetic energy and the power of the viscous stress vary between two channel sections and the flow is neither dynamically nor thermally fully-developed. Thus, from Eq. (20), the sum of the 
source terms, $P W+V D$, and the sum $C O N V+D I F F$ are not zero and are never equal to $V S P$. Consequently, contrary to the previously considered incompressible cases, DIFF does not converge to $V S P$ with the grid size after the thermal entrance zone (see the zoom in Fig. $14)$.

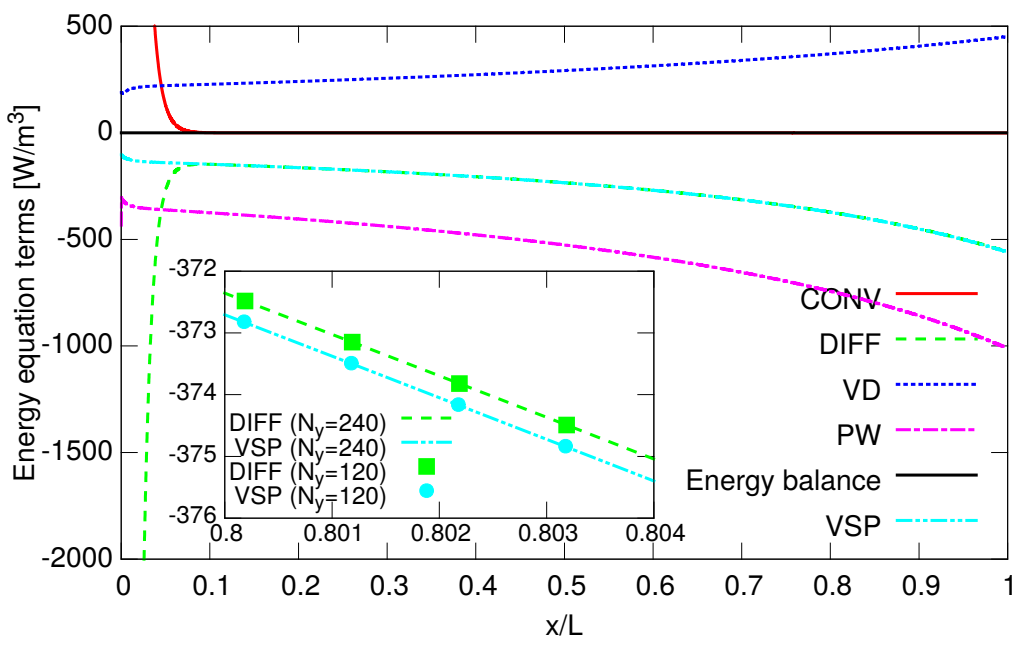

Fig. 14: Streamwise profiles of the thermal energy balance $(C O N V+D I F F-P W-V D)$, of its different terms and of the power of the viscous stress at the wall $(V S P)$, for the compressible slipping flow at $D_{h}=6 \mu \mathrm{m}, p_{\text {in }}=0.2 \mathrm{bar}, p_{\text {out }}=0.1$ bar (see Table 5 ).

In conclusion, for compressible flows in isothermal wall channels, the total wall heat flux is not null because the power of the viscous stress does not compensate for the diffusive flux. Therefore $N u_{t}=N u_{\text {diff }}+N u_{V S P}$ is not zero everywhere along the walls. This is illustrated for instance in Fig. 7, for the compressible case (b) of Table 2, where the total local Nusselt number increases downstream due to the gas expansion and its cooling in the channel core. However, when the hydraulic diameter is very small and the pressure difference between the inlet and the outlet of the channel is moderate, the Mach number generally remains small. The source term variation has almost no effect on the Nusselt number, except in the thermal entrance zone and just upstream the outlet where the gas expansion can be locally more important. This is illustrated on Fig. 15 which presents the streamwise profiles of the total Nusselt number, $N u_{t}$, for the weakly compressible flows of Table 5 . Since the temperature difference at the inlet is $T_{w}-T_{\text {in }}=30 \mathrm{~K}$, the Nusselt number reaches at first a plateau for $0.01 \lesssim \frac{x}{L} \lesssim 0.05$, where $2 \lesssim N u \lesssim 4$. This first plateau corresponds to the zone where the CONV and DIFF terms of the energy balance dominate the $P W$ and $V D$ source terms (see Fig. 14). Then $N u_{t}$ steeply decreases for $0.05 \lesssim \frac{x}{L} \lesssim 0.2$ to reach a second plateau where $N u_{t}<5 \times 10^{-4}$, except close to the channel outlet where the cooling due to gas expansion (at $p_{a v}=0.1$ bar) and the outlet boundary conditions disrupt the Nusselt number variation. This second plateau corresponds to the zone where the $C O N V$ and $D I F F$ terms reach very small values, i.e. much smaller than the $P W$ and $V D$ values in the energy balance (see Fig. 14). Therefore, when $D_{h}\left(\right.$ or $\left.R e_{D h}\right)$ is small, $N u_{t}$ is not null but keeps very small values along the walls, at least in the central zone of the channel, far enough from the inlet and outlet sections. 


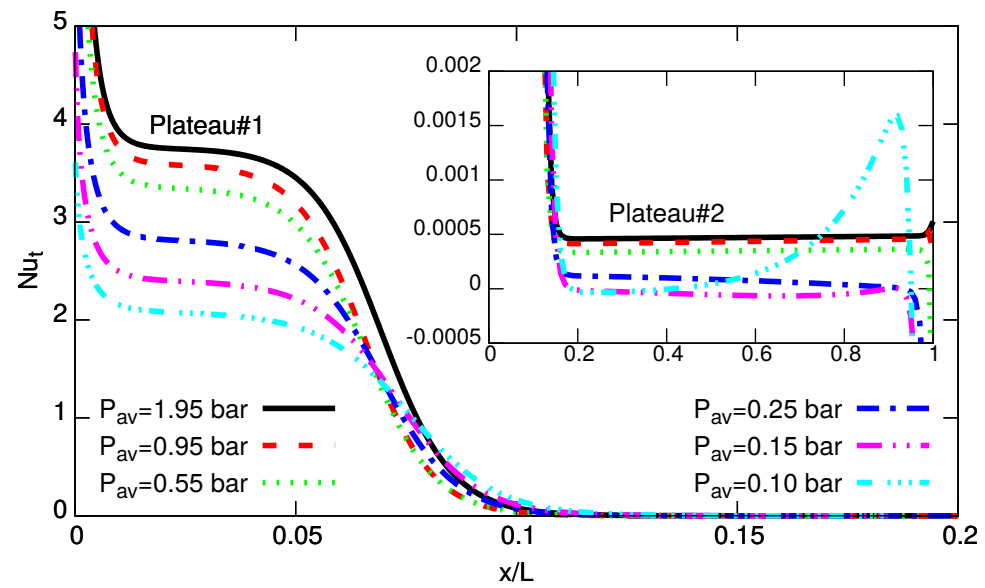

Fig. 15: Streamwise profiles of the total Nusselt number, $N u_{t}(X)$, for the weakly compressible micro-flows of Table 5 , at $D_{h}=6 \mu \mathrm{m}, \Delta p_{\text {ref }}=0.1$ bar and a decreasing average pressure.

The integral mean of the total Nusselt number along the whole wall, $\overline{N u_{t}}$, and along the wall part corresponding to the second $N u$-plateau, $\overline{N u_{t}}$,plateau\#2, are plotted on Fig. 16 with respect to the average Knudsen number, $K n_{a v}=\left(K n_{\text {in }}+K n_{\text {out }}\right) / 2$, for the weakly compressible microflows of Table 5. Due to the influence of the first $N u$-plateau close to the entrance, the $\overline{N u_{t}}$ values are at least three orders of magnitude higher than the $\overline{N u_{t}, p l a t e a u \# 2}$ values. $\overline{N u_{t}}$ decreases with respect to $K n_{a v}$ according to the correlation: $\overline{N u_{t}}=0.113 K n_{a v}^{-0.184}$. $\overline{N u}_{t}$,plateau\#2 also decreases with $K n_{a v}$ but no clear correlation can be found.



Fig. 16: Integral mean of the total Nusselt number along the whole wall $\left(\overline{N u_{t}}\right)$ and along the wall part corresponding to the second $N u$-plateau $\left(\overline{N u_{t}, p l a t e a u \# 2}\right)$, with respect to the average Knudsen number, for the weakly compressible micro-flows of Table 5 , at $D_{h}=$ $6 \mu \mathrm{m}, \Delta p_{\text {ref }}=0.1$ bar and a decreasing average pressure.

In the micro tube experiments by Demsis et al. [23,24], a linearly decreasing slope in LogLog scale of the relation $\overline{N u_{t}}=f\left(K n_{a v}\right)$ is also observed. However, in these experiments, the values of the average Nusselt number, $\overline{N u_{t}}$, along the whole wall vary between $3 \times 10^{-4}$ and 
$2 \times 10^{-2}$ while they vary between 0.16 and 0.3 in our simulations (Fig. 16). It is clear from Fig. 15 that our values of $\overline{N u_{t}}$ are highly influenced by the thermal entrance zone of length $x=0.1 L=5 H=2.5 D_{h}$ where the Nusselt number is larger. If the channel aspect ratio was much larger than the one used $(L / H=50)$, the $\overline{N u_{t}}$ numerical values would be much smaller. However in the experiments $[23,24]$, the channel aspect ratio is also relatively small $(L / D \approx 40$, with $D$ the tube diameter). Therefore, it looks like as only the central part of the duct with low Nusselt numbers be selected for the calculations of the average Nusselt numbers in [23,24]. This could be an explanation for the small Nusselt number values obtained. In these experiments, the temperature probes were indeed shifted $1.4 \mathrm{D}$ downstream the inlet and $1.4 \mathrm{D}$ upstream the outlet of the inner tube of the heat exchanger. Furthermore, due to the heat exchanger design, the small values of the Reynolds number and the likely presence of inverse diffusion, the inlet and outlet thermal conditions were maybe not perfectly controlled (read the criticism in [71]).

\section{Conclusion}

The thermal aspects of the modeling of weakly rarefied gaseous flows $(0.001<K n \leq 0.1)$ with first-order slip and temperature jump models have been discussed in details thanks to a dimensional analysis, an asymptotic analysis and numerical simulations. This model has been analyzed in the case of the forced convection of a cold gas flowing in long flat micro channels isothermally heated. The order of magnitude of the pressure work $(P W)$ and viscous dissipation $(V D)$ in the bulk flow and the order of magnitude of the power of the viscous stress $(V S P)$ and thermal creep $(T C)$ at the walls have been determined with respect to the flow parameters $\left(R e_{w}, M a_{w}, K n_{w}\right)$ and $\epsilon=D_{h} / L$.

The main remarks and tricky points highlighted are:

- in the wall boundary conditions, the values of the viscous, thermal slip and temperature jump coefficients must be all compatible with the same characteristics: a diffusive or fully accommodating wall for instance (see [4]);

- modeling the Knudsen layer requires to introduce a temperature jump, even if a heat flux is imposed at the wall;

- computing the total heat flux at the wall requires to take into account the power of the viscous stress at the wall $(V S P)$;

- the integral $P W+V D$ of the pressure work and viscous dissipation in a channel slice is approximately equal to the power of the viscous stress at the wall. Therefore, $V S P$ cannot be neglected in the energy flux if $P W+V D$ is not negligible;

- for the "fully-developed" weakly rarefied gas flows in isothermally heated micro-channels considered in the present paper, the viscous dissipation, the pressure work and the viscous stress power at the walls cannot be neglected in the mathematical model while the thermal creep is negligible far from the entrance.

In the second part of the paper, the analytical asymptotic solutions of the flow and thermal fields established by Arkilic et al. [62] and Cai et al. [63] for forced convection of gas in isothermal wall micro-channels of large aspect ratio are extended to compressible flows and the Nusselt number computation (see Eqs. (54)-(66) in $§ 4$ and Appendix B). It is shown that the local total 
Nusselt number, $N u_{t}(x)$, tends towards zero, at least at first order with $\epsilon$, far enough from the thermal entrance zone, provided that the contribution of the power of the viscous stress at the wall is accounted for.

This analytical solution, valid at low Reynolds and Mach numbers $\left(R e_{w} \lesssim O(1)\right.$ and $M a_{w}<$ $O(1)$ ), is compared with the numerical solution of the full model by a finite volume method. This numerical solution takes into account the first order slip and thermal jump conditions at the walls, the power of the viscous stress at the wall, the pressure work, the viscous dissipation and the thermal creep term. In the simulated cases reported in Table 2, the analytical and numerical solutions agree well for a wide range of flow parameters and flow types.

Finally, a thorough analysis of the streamwise distribution of the different contributions to the thermal and mechanical energy balances and to the total heat flux is carried out in the cases of non slipping or slipping, incompressible and compressible flows in isothermal wall micro-channels. It is shown that the contribution of the power of the viscous stress at the walls must not be neglected because it is opposite to and of the order of magnitude of the diffusive flux at the walls. Furthermore, the total wall heat flux and total Nusselt number tend towards zero all the more so as the Knudsen number is large (significant rarefaction effects) and the Reynolds number is small (short thermal entrance zone). This is coherent with the Nusselt number values obtained in the experiments by Demsis et al. [23,24]. 


\section{Appendix A: order of magnitude of the different terms linked with heat transfer in long micro channels}

The order of magnitude of the different terms linked with heat transfer in the conservation equations and boundary conditions are evaluated as a function of the rarefaction and compressibility intensities, represented by $K n_{w}$ and $M a_{w}$, respectively. The study is limited to subsonic low rarefied gas flows: $\operatorname{Pr} \sim 1, M a_{w}<1$ and $10^{-3} \lesssim K n_{w} \lesssim 0.1$. The typical micro-flows considered concern gas flows through long micro channels of hydraulic diameter $D_{h} \sim 1$ to $10 \mu \mathrm{m}$ and length $L \sim 0.1$ to $10 \mathrm{~mm}$. The inverse of the longitudinal aspect ratio is therefore a small parameter: $10^{-4} \lesssim \epsilon=\frac{D_{h}}{L} \lesssim 0.1$.

Using Eqs. (49), (50) and (51), the order of magnitude of $R e_{w}, \frac{1}{\operatorname{Pr}_{w} \operatorname{Re}_{w}} \frac{\bar{u}_{r e f}}{u_{r e f}}, \epsilon \operatorname{Pr}_{w} K n_{w}^{2} R e_{w} \frac{u_{r e f}}{\bar{u}_{r e f}}$, $\frac{\Delta T_{r e f}}{T_{w}}$ and $\frac{\Delta p_{r e f}}{p_{a v}}$ that appear in the conservation equations (39)-(41), state equation (43) and boundary conditions (44)-(47) can be computed with respect to the orders of magnitude of $M a_{w}$ and $K n_{w}$. The orders of magnitude of all these linked parameters are given in Table 6 . They are expressed as a function of $\epsilon$ only. To this end, it was considered that $\frac{\bar{u}_{\text {ref }}}{u_{r e f}} \sim \epsilon^{1 / 2}$ for $10^{-3} \lesssim \epsilon \lesssim 10^{-2}$ since, from Eq. (48), $\frac{1}{48} \leq \bar{u}_{\text {ref }} / u_{\text {ref }} \leq \frac{1}{22}$ for $0 \leq K n_{w} \leq 0.1$ and $\sigma_{\mu}=1$.

\begin{tabular}{|cc|c|c|c|}
\cline { 3 - 5 } \multicolumn{1}{c|}{} & \multicolumn{3}{c|}{$M a_{w}$} \\
\hline \multirow{2}{*}{\multicolumn{1}{c|}{$e_{w}$}} & $\epsilon^{2}$ & $\epsilon$ & 1 \\
\hline$\epsilon_{w}$ & 1 & $\epsilon^{-1}$ & $\epsilon^{-2}$ \\
\cline { 2 - 5 } & $\epsilon$ & $\epsilon$ & 1 & $\epsilon^{-1}$ \\
\hline$\epsilon^{1 / 2}$ & $\epsilon^{1.5}$ & $\epsilon^{0.5}$ & $\epsilon^{-0.5}$ \\
\hline
\end{tabular}

(a) $R e_{w}=\sqrt{\frac{\pi \gamma}{2}} M a_{w} / K n_{w} \sim M a_{w} / K n_{w}$ (Eq. (49))

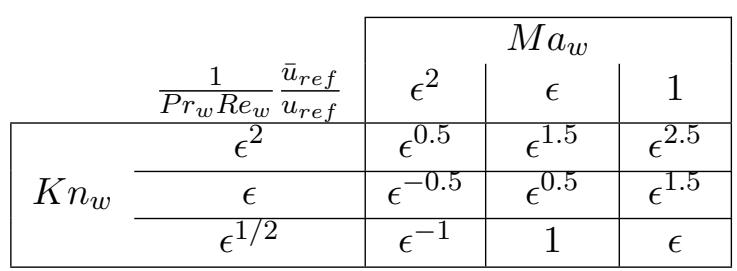

(b) Order of magnitude of $P W$ and $V D$ in Eq. (41): $\frac{1}{P r_{w} R_{w}} \frac{\bar{u}_{r e f}}{u_{r e f}}=\sqrt{\frac{2}{\pi \gamma}} \frac{K n_{w}}{M a_{w} P r_{w}} \frac{\bar{u}_{r e f}}{u_{r e f}} \sim \frac{K n_{w}}{M a_{w}} \epsilon^{1 / 2}$

\begin{tabular}{|cc|c|c|c|}
\cline { 3 - 5 } \multicolumn{1}{c|}{} & \multicolumn{1}{c}{} & \multicolumn{3}{c|}{$M a_{w}$} \\
\hline \multirow{3}{*}{$K n_{w} K n_{w}^{2} R e_{w} \frac{u_{\text {ref }}}{\bar{u}_{\text {ref }}}$} & $\epsilon^{2}$ & $\epsilon$ & 1 \\
\cline { 2 - 5 } & $\epsilon^{2}$ & $\epsilon^{4.5}$ & $\epsilon^{3.5}$ & $\epsilon^{2.5}$ \\
\cline { 2 - 5 } & $\epsilon$ & $\epsilon^{3.5}$ & $\epsilon^{2.5}$ & $\epsilon^{1.5}$ \\
\hline$\epsilon^{1 / 2}$ & $\epsilon^{3}$ & $\epsilon^{2}$ & $\epsilon$ \\
\hline
\end{tabular}

(c) Order of magnitude of 5 to $10 \times T C$ in Eq. (44): $\epsilon \operatorname{Pr}_{w} K n_{w}^{2} R e_{w} \frac{u_{r e f}}{\bar{u}_{r e f}} \sim \epsilon^{1 / 2} M a_{w} K n_{w}$

\begin{tabular}{|cc|c|c|c|}
\cline { 3 - 5 } \multicolumn{1}{c|}{} & \multicolumn{3}{c|}{$M a_{w}$} \\
\hline \multirow{2}{*}{} & $\frac{\Delta T_{\text {ref }}}{T_{w}}$ & $\epsilon^{2}$ & $\epsilon$ & 1 \\
\hline$\epsilon^{2}$ & $\epsilon_{w}$ & $\epsilon$ & $\epsilon^{-1}$ \\
\cline { 2 - 5 } & $\epsilon$ & $\epsilon^{3}$ & $\epsilon$ & $\epsilon^{-1}$ \\
\cline { 2 - 5 } & $\epsilon^{1 / 2}$ & $\epsilon^{3}$ & $\epsilon$ & $\epsilon^{-1}$ \\
\hline
\end{tabular}

(d) $\frac{\Delta T_{r e f}}{T_{w}}=(\gamma-1) \operatorname{Pr}_{w}\left(M a_{w} \frac{u_{r e f}}{\bar{u}_{\text {ref }}}\right)^{2} \sim$ $\epsilon^{-1} M a_{w}^{2}$ (Eq. (51))

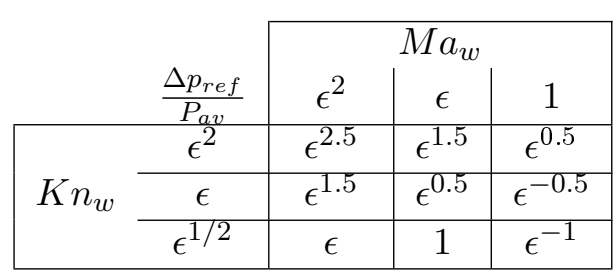

(e) $\frac{\Delta p_{r e f}}{P_{a v}}=\frac{\gamma}{\epsilon} \frac{M a_{w}^{2}}{R e_{w}} \frac{u_{r e f}}{\bar{u}_{\text {ref }}} \sim \epsilon^{-3 / 2} M a_{w} K n_{w}$ (Eq. (50))

Tab. 6: Orders of magnitude of the dimensionless groups that appear in the equations and boundary conditions of the model, with respect to $M a_{w}$ and $K n_{w}$ expressed as a function of $\epsilon$. 
First, in the momentum and energy conservation equations (39)-(41), the advection and transport terms are of the order of $O(\epsilon)$. Furthermore, it can be checked from Tables $6 \mathrm{a}$ and $6 \mathrm{~b}$ that all the other terms of these equations are at least of the order of $O\left(\epsilon^{0.5}\right)$ when $\operatorname{Re}_{w} \lesssim O(1)$ and $M a_{w}<O(1)$. Therefore the advection and transport terms terms are negligible with respect to the other terms in this parameter range. Since the analytical solutions are established by neglecting these terms, they are thus valid for $R e_{w} \lesssim O(1)$ and $M a_{w}<O(1)$. In this parameter range, Tables $6 \mathrm{~d}$ and $6 \mathrm{e}$ indicate that the relative temperature and pressure variations keep small : $\frac{\Delta T_{r e f}}{T_{w}} \lesssim O(\epsilon)$ and $\frac{\Delta p_{r e f}}{P_{a v}} \lesssim O(1)$.

The pressure work $(P W)$ and the viscous dissipation $(V D)$ in the energy equation (41) are of the order of $\frac{1}{P r_{w} R e_{w}} \frac{\bar{u}_{r e f}}{u_{r e f}} \sim \frac{K n_{w}}{M a_{w}} \epsilon^{1 / 2}$ (see Table $6 \mathrm{~b}$ ). As a consequence $P W$ and $V D$ cannot be neglected in the energy equation whatever the values of $M a_{w}$ and $K n_{w}$. They are indeed at least of the order of the transverse diffusion term $\left(\sim \frac{1}{P r_{w} R e_{w}} \frac{\bar{u}_{r e f}}{u_{r e f}}\right)$ or of the transport term $(\sim \epsilon)$. Similarly, the power of the viscous stress in the expression of the wall heat flux (second R.H.S term in Eq. (47)) is of the order of the diffusive term (first R.H.S term), independently of $K n_{w}$ and $M a_{w}$. Consequently, $N u_{V S P}$ in Eqs. (64) and (93) is never negligible.

The thermal creep (TC) term (second R.H.S term in Eq. (44)) is of the order of $\epsilon \operatorname{Pr}_{w} K n_{w}^{2} R e_{w} \frac{u_{r e f}}{\bar{u}_{r e f}}$ with a quite small factor ahead: $\sigma_{\theta} \frac{2(\gamma-1)}{\pi \gamma} \sim 0.1$ to 0.2 . As for the velocity slip term (first R.H.S term of (44)), it is of the order of $K n_{w}$. Therefore, from Table 6c, the thermal creep term appears to be always negligible, whatever $M a_{w}$ and $K n_{w}$, for the developed micro-flows considered here. This conclusion is obviously not valid when the entrance effects are important: in this case, the scale for the temperature difference should be taken as $\Delta T_{r e f}=T_{w}-T_{i n}$. Then it can be shown that the $T C$ term must be taken into account at small Mach numbers $\left(M a_{w} \lesssim \epsilon^{2}\right)$ for $\frac{\Delta T_{r e f}}{T_{i n}} \sim 1$, which corresponds to small Reynolds numbers $\left(R e_{w} \lesssim 1\right)$.

\section{Appendix B: asymptotic solutions for long micro channels with isothermal walls}

Following the asymptotic analyzes by Arkilic et al. [62] and Cai et al. [63], considering weakly rarefied flows with small temperature variations in long micro-channels $\left(\epsilon=D_{h} / L<<1\right)$, the dimensionless quantities can be written as asymptotic expansions in $\epsilon$ :

$$
\begin{array}{rll}
U=U_{0}+\epsilon U_{1}+\epsilon^{2} U_{2}+\ldots ; & V=\epsilon V_{1}+\epsilon^{2} V_{2}+\ldots ; & P=P_{0}+\epsilon P_{1}+\epsilon^{2} P_{2}+\ldots \\
\theta=\epsilon \theta_{1}+\epsilon^{2} \theta_{2}+\ldots ; & \rho^{*}=\rho_{0}^{*}+\epsilon \rho_{1}^{*}+\epsilon^{2} \rho_{2}^{*}+\ldots ; & \mu^{*}=\mu_{0}^{*}+\epsilon \mu_{1}^{*}+\epsilon^{2} \mu_{2}^{*}+\ldots ; \text { etc. }
\end{array}
$$

Note that $V_{0}=0$ because the continuity equation (38) gives $\frac{\partial \rho_{0}^{*} V_{0}}{\partial Y}=0$ at the zero order with $\epsilon$ and $V_{0}=0$ at the wall. At the zero order with $\epsilon$, the dimensionless temperature is also supposed constant, equal to $\theta_{0}=0$, i.e. $T=T_{w}$. The temperature expansion is thus compatible with walls at constant or weakly varying temperature.

Then, the dimensionless conservation equations (38)-(41) can be written at the zero or first order with $\epsilon$ accounting for the ranges of variations of the dimensionless parameters expressed as a function of $\epsilon$ in Table 6 of Appendix A. When $R e_{w} \lesssim O\left(\epsilon^{-2}\right)$ (maximum Reynolds number in the present study; see Table 6a), the inertial and viscous terms of the spanwise momentum equation (40) are negligible and the lowest order with $\epsilon$ gives: 


$$
\frac{\partial P_{0}}{\partial Y}=0 \Longleftrightarrow P_{0}=P_{0}(X)
$$

For $R e_{w} \lesssim O\left(\epsilon^{-1}\right)$, we also get $P_{1}=P_{1}(X)$. At first order, the continuity equation (38) is:

$$
\frac{\partial \rho_{0}^{*} U_{0}}{\partial X}+\frac{\partial \rho_{0}^{*} V_{1}}{\partial Y}=0
$$

and by noting $P_{0}^{*}(X)=1+\frac{\Delta p_{r e f}}{p_{a v}} P_{0}(X)$, the ideal gas law (43) at the zero order with $\epsilon$ reads:

$$
\rho_{0}^{*}(X)=1+\frac{\Delta p_{\text {ref }}}{p_{\text {av }}} P_{0}(X)=P_{0}^{*}(X)
$$

Then Eq. (74) at first order with $\epsilon$ can also be written:

$$
\frac{\partial P_{0}^{*} U_{0}}{\partial X}+\frac{\partial P_{0}^{*} V_{1}}{\partial Y}=0
$$

For $R e_{w} \lesssim O(1)$ and $M a_{w}<O(1)$, at the lowest order with $\epsilon$, the streamwise momentum equation (39) becomes:

$$
-\frac{\partial P_{0}}{\partial X}+\frac{\partial}{\partial Y}\left(\mu_{0}^{*} \frac{\partial U_{0}}{\partial Y}\right)=0
$$

and, taking into account the continuity equation (74), the energy equation (41) writes:

$$
\frac{\partial}{\partial Y}\left(k_{0}^{*} \frac{\partial \theta_{1}}{\partial Y}\right)=-\frac{1}{\epsilon}\left[U_{0} \frac{\partial P_{0}}{\partial X}+\mu_{0}^{*}\left(\frac{\partial U_{0}}{\partial Y}\right)^{2}\right]
$$

Thus the analytical solutions of micro-flows at $R e_{w} \lesssim O(1)$ and $M a_{w}<O(1)$ can be computed from the asymptotic continuity equation (76), streamwise Navier-Stokes equation (77) and energy equation (78). The boundary conditions for $U_{0}, V_{0}, V_{1}, P_{0}$ and $\theta_{1}$ are the same as for $U, V, P$ and $\theta$, with a negligible thermal creep term in Eq. (44) (see Appendix A):

- on the entrance, at $X=0, \forall Y \in\left[0, \frac{1}{4}\right], P_{0, i n}=\frac{1}{2}, \frac{\partial U_{0}}{\partial X}=0, V_{1, i n}=0$ and $\theta_{1, i n}=\frac{T_{i n}-T_{w}}{\epsilon \Delta T_{r e f}}$;

- on the outlet, at $X=1, \forall Y \in\left[0, \frac{1}{4}\right], P_{0, \text { out }}=-\frac{1}{2}, \frac{\partial U_{0}}{\partial X}=\frac{\partial V_{1}}{\partial X}=\frac{\partial \theta_{1}}{\partial X}=0$;

- on the channel axis, at $Y=0, \forall X \in[0,1], \frac{\partial U_{0}}{\partial Y}=V_{1}=\frac{\partial \theta_{1}}{\partial Y}=0$;

- on the wall, at $Y=\frac{1}{4}, \forall X \in[0,1]$, the velocity boundary conditions are:

$$
\begin{gathered}
U_{0, g}=\left.\frac{-\sigma_{\mu} K n_{w} \mu_{0, g}^{*}}{1+\frac{\Delta p_{r e f}}{p_{a v}} P_{0, g}} \frac{\partial U_{0}}{\partial Y}\right|_{g} \\
V_{1, g}=0
\end{gathered}
$$

The thermal boundary conditions in the case of a uniform wall temperature $T_{w}$ read:

$$
\theta_{1, g}=\left.\frac{-\xi_{T} K n_{w} \mu_{0, g}^{*}}{1+\frac{\Delta p_{r e f}}{p_{\text {in }}} P_{0, g}} \frac{\partial \theta_{1}}{\partial Y}\right|_{g}
$$


and, from Eq. (47), the dimensionless total wall heat flux writes:

$$
q_{t, w}^{*}=\frac{q_{w} D_{h}}{k_{w} \Delta T_{r e f}}=\left.k_{0, g}^{*} \epsilon \frac{\partial \theta_{1}}{\partial Y}\right|_{g}+\left.\mu_{0, g}^{*} U_{0, g} \frac{\partial U_{0}}{\partial Y}\right|_{g}
$$

The analytical solution of this asymptotic model can easily be computed if the physical properties are considered constant and equal to their values at $T_{w}: \mu_{0}^{*}=1$ and $k_{0}^{*}=1$. In this case, for a uniform wall temperature $T_{w}$, this analytical solution, adapted from Arkilic et al. [62] and Cai et al. [63], is given by the equations (54)-(62) in $\S 4$.

Temperature jump at the wall: $\epsilon \theta_{1, g}(X)$

The temperature jump at the wall is equal to $\theta_{g} \approx \epsilon \theta_{1, g}$ with:

$$
\epsilon \theta_{1, g}(X)=\epsilon \theta_{1}\left(X, Y=\frac{1}{4}\right)=\frac{-A \cdot B}{16} \frac{F(X)}{P_{0}^{* 2}(X)}=-\frac{\sigma_{\mu} \xi_{T}}{16} K n_{w}^{2}\left(\frac{1}{P_{0}^{*}(X)} \frac{d P_{0}(X)}{d X}\right)^{2}
$$

The fluid temperature close to the wall is thus different from the wall temperature $\left(\theta_{1, g} \neq 0\right)$ when $K n_{w} \neq 0$.

\section{Average streamwise velocity: $\overline{U_{0}}(X)$}

The average velocity is defined by:

$$
\overline{U_{0}}(X)=4 \int_{Y=0}^{1 / 4} U_{0}(X, Y) d Y=-2 \frac{d P_{0}}{d X} \int_{Y=0}^{1 / 4}\left[b(X)-Y^{2}\right] d Y
$$

Therefore:

$$
\begin{gathered}
\overline{U_{0}}(X)=\frac{1-48 b(X)}{96} \frac{d P_{0}(X)}{d X} \\
\frac{U_{0}(X, Y)}{\overline{U_{0}}(X)}=\frac{b(X)-Y^{2}}{b(X)-\frac{1}{48}}
\end{gathered}
$$

Mass flow rate: $\dot{m}$

The inlet and outlet pressures, $p_{\text {in }}$ and $p_{\text {out }}$, being inputs of the problem formulation, the mass flow rate, $\dot{m}$, is an output of the calculations. An approximate analytical expression of $\dot{m}$ as a function of $p_{\text {in }}$ and $p_{\text {out }}$ can be established, at zero order with $\epsilon$, starting from its definition:

$$
\dot{m}=2 \int_{y=0}^{H / 2} \rho(x, y) u(x, y) d y=4 \rho_{w} u_{r e f} H \int_{Y=0}^{1 / 4} \rho^{*}(X, Y) U(X, Y) d Y
$$

Then, using Eq. (75), $\dot{m}$ can be written: 


$$
\dot{m}=4 \rho_{w} u_{r e f} H \rho_{0}^{*}(X) \int_{Y=0}^{1 / 4} U_{0}(X, Y) d Y=\rho_{w} u_{r e f} H P_{0}^{*}(X) \overline{U_{0}}(X)
$$

From Eqs. (35), (56) and (85), the following expression is derived:

$$
\dot{m}=\frac{H^{3}}{24 L \mu_{w}} \frac{1}{r T_{w}}\left(p_{\text {in }}^{2}-p_{\text {out }}^{2}\right)\left[1+12 \sigma_{\mu} K n_{w}\right]=\rho_{w} u_{r e f} H \frac{1+12 \sigma_{\mu} K n_{w}}{48}
$$

The expression (89) is similar to that derived by Arkilic et al. [62] for an isothermal weakly compressible flow. It can be deduced from Eqs. (88) and (89) that:

$$
4 \rho_{0}^{*}(X) \int_{Y=0}^{1 / 4} U_{0}(X, Y) d Y=\rho_{0}^{*}(X) \overline{U_{0}}(X)=P_{0}^{*}(X) \overline{U_{0}}(X)=\frac{1+12 \sigma_{\mu} K n_{w}}{48}
$$

Wall Nusselt number: $N u_{t}(X)$

For a constant wall temperature $T_{w}$, the total wall Nusselt number can be defined as:

$$
N u_{t}(x)=\frac{q_{t, w} D_{h}}{k_{w}\left(T_{w}-\bar{T}(x)\right)}=\frac{-q_{t, w} D_{h}}{k_{w} \Delta T_{r e f} \bar{\theta}(X)}
$$

where $q_{t, w}$ is given by Eq. (19). The dimensionless form of the bulk temperature (Eq. (65)) reads:

$$
\bar{\theta}(x)=\frac{\bar{T}(x)-T_{w}}{\Delta T_{r e f}}=\frac{2}{\dot{m}} \int_{0}^{H / 2} \rho(x, y) u(x, y) \theta(x, y) d y
$$

At the first order of the temperature, $\bar{\theta}(X) \approx \epsilon \overline{\theta_{1}}(X)$. Then, using Eq. (82) with constant physical properties, the total local Nusselt number, defined by Eq. (64), also writes:

$$
N u_{t}(x)=N u_{d i f f}+N u_{V S P}=\frac{-1}{\epsilon \overline{\theta_{1}}(X)}\left(\left.\epsilon \frac{\partial \theta_{1}}{\partial Y}\right|_{g}+\left.U_{0, g} \frac{\partial U_{0}}{\partial Y}\right|_{g}\right)
$$

The three terms involved in Eq. (93) are calculated below:

- The temperature first derivative at the walls is obtained from Eq. (57) for $\theta_{1}(X, Y)$. Thus, using Eq. (59), the conductive heat flux close to the walls is:

$$
\left.\epsilon \frac{\partial \theta_{1}}{\partial Y}\right|_{g}=F(X) \frac{16 b(x)-1}{2}=\frac{F(X)}{2} \frac{A}{P_{0}^{*}(X)}
$$

- The viscous stress power at the walls is computed from Eq. (54) for $U_{0}(X, Y)$ :

$$
\left.U_{0, g} \frac{\partial U_{0}}{\partial Y}\right|_{g}=\left.\frac{-1}{2}\left(\frac{d P_{0}(X)}{d X}\right)^{2}\left[b(X)-Y^{2}\right] Y\right|_{g}=\frac{-1}{128}\left(\frac{d P_{0}(X)}{d X}\right)^{2}[16 b(X)-1]
$$


From the definitions of $F(X)$ and $b(X)$ (Eqs. (58) and (59)), we obtain:

$$
\left.U_{0, g} \frac{\partial U_{0}}{\partial Y}\right|_{g}=-\frac{F(X)}{2} \frac{A}{P_{0}^{*}(X)}
$$

- From the definition (92) of the average bulk temperature, using Eqs. (86) and (90), the first order approximation of $\bar{\theta}(X)$ becomes:

$$
\overline{\theta_{1}}(X)=\frac{4}{\overline{U_{0}}(X)} \int_{Y=0}^{1 / 4} U_{0}(X) \theta_{1}(X, Y) d Y=\frac{192}{48 b(X)-1} \int_{Y=0}^{1 / 4}\left(b(X)-Y^{2}\right) \theta_{1}(X, Y) d Y
$$

Using Eq. (57) for $\theta_{1}(X, Y)$ leads to:

$$
\overline{\theta_{1}}(X)=\frac{-192}{48 b(X)-1} \frac{F(X)}{\epsilon} \int_{Y=0}^{1 / 4}\left(b(X)-Y^{2}\right)\left\{8 Y^{4}-16 b(X) Y^{2}+J(X)\right\} d Y
$$

and, after integration, we get:

$$
\epsilon \overline{\theta_{1}}(X)=F(X) \frac{17920 b(X)^{2}-1008 b(X)+15+J(X)(1120-53760 b(X))}{1120(48 b(X)-1)}
$$

For $K n_{w}=0$ (no slip and no rarefaction case ), $b=\frac{1}{16}, J=\frac{1}{32}$ and $\epsilon \overline{\theta_{1}}(X)=\frac{3 F(X)}{140}$.

The viscous stress power at the wall (Eq. (95)) is thus the opposite of the conduction heat flux at the wall (Eq. (94)). Consequently, using Eq. (98), the total wall Nusselt number (93) is:

$$
N u_{t}(X)=\frac{-560(48 b(X)-1)}{17920 b(X)^{2}-1008 b(X)+15+J(X)(1120-53760 b(X))}\left(\frac{A}{P_{0}^{*}(X)}-\frac{A}{P_{0}^{*}(X)}\right)=0
$$

Since $b(X)>0$ and $J(X)>0$ whatever $X \in[0,1]$, the Nusselt number denominator never tends towards zero and $N u_{t}(X)=0$ everywhere at the first order with $\epsilon$.

From Eqs. (94) and (95), it appears that the conduction heat flux and the viscous stress power at the walls both vanish when $K n_{w}=0$ since then $A=0$ (see Eq. (61)). On the other hand, for $K n_{w} \neq 0$, the conduction heat flux at the walls is different from zero but the total heat flux at the walls and thus the total Nusselt number tend everywhere towards zero, at least at the first order with $\epsilon$. These results are linked to the approximations used in deriving the analytical solution: the dynamical and thermal entrance effects are indeed neglected. In particular, the convective term and the streamwise variation of the temperature from inlet are not accounted for in the energy equation (78). This approximation is justified in the case of long micro-channel because the Reynolds number is generally small and the thermal entrance length is thus very short. 


\section{References}

[1] S.G. Kandlikar, S. Colin, Y. Peles, S. Garimella, R.F. Pease, J.J. Brandner, and D.B. Tuckerman. Heat transfer in microchannels -2012 status and research needs. J. Heat Transf., 135(9) (2013) 091001.

[2] M. Gad-el Hak. Fluid mechanics of microdevices - The Freeman scholar lecture. J. Fluids Engin., 121(1) (1999) 5-33.

[3] G.E. Karniadakis, A. Beskok, and N.R. Aluru. Microflows and Nanoflows - Fundamentals and Simulation, volume 29 of Interdisciplinary Applied Mathematics, Springer New York, 2005 .

[4] F. Sharipov, Data on the velocity slip and temperature jump on a gas-solid interface, Journal of Physical and Chemical Reference Data, 40(2) (2011) 023101.

[5] D.A. Lockerby, J.M. Reese, and M.A. Gallis, The usefulness of higher-order constitutive relations for describing the Knudsen layer, Phys. Fluids, 17(10) (2005) 100609.

[6] Y. Yang, G.L. Morini, and J.J. Brandner, Experimental analysis of the influence of wall axial conduction on gas-to-gas micro heat exchanger effectiveness. Int. J. Heat Mass Transf., 69 (2014) 17-25.

[7] T. Dixit and I. Ghosh. Review of micro- and mini-channel heat sinks and heat exchangers for single phase fluids, Renewable and Sustainable Energy Reviews, 41(0) (2015) 1298-1311.

[8] N. K. Gupta and Y. B. Gianchandani, Porous ceramics for multistage Knudsen micropumpsmodeling approach and experimental evaluation, J. Micromech. Microengin., 21(9) (2011) 095029 .

[9] N. K. Gupta and Y. B. Gianchandani, Thermal transpiration in mixed cellulose ester membranes: Enabling miniature, motionless gas pumps, Microporous and Mesoporous Materials, 142(2-3) (2011) 535-541.

[10] N. K. Gupta, S. An, and Y. B. Gianchandani, A Si-micromachined 48-stage Knudsen pump for on-chip vacuum, J. Micromech. Microengin., 22(10) (2012) 105026.

[11] S. Nakaye, H. Sugimoto, N. K. Gupta, and Y. B. Gianchandani, Thermally enhanced membrane gas separation, European Journal of Mechanics-B/Fluids, 49(A) (2015) 36-49.

[12] Y. Qin and Y. B. Gianchandani, iGC1: An integrated fluidic system for gas chromatography including Knudsen pump, preconcentrator, column, and detector microfabricated by a three-mask process, J. Microelectromech. Syst., 23(4) (2014) 980-990.

[13] Y. Qin and Y. B. Gianchandani, iGC2: An architecture for micro gas chromatographs utilizing integrated bi-directional pumps and multi-stage preconcentrators, J. Micromech. Microengineering, 24(6) (2014) 065011. 
[14] G. Manzoni, Micro and nanotechnology applications for space micropropulsion. In Micromanufacturing and Nanotechnology, Springer Berlin Heidelberg, 2006, pp 197-218.

[15] F. La Torre, S. Kenjeres, J.-L. Moerel, and C.R. Kleijn, Hybrid simulations of rarefied supersonic gas flows in micro-nozzles, Computers and Fluids, 49(1) (2011) 312-322.

[16] W.F. Louisos, D.L. Hitt, Analysis of transient flow in supersonic micronozzles, Journal of Spacecraft and Rockets, 48(2) ( 2011) 303-311.

[17] W.F. Louisos, D.L. Hitt, Influence of wall heat transfer on supersonic micronozzle performance, Journal of Spacecraft and Rockets, 49(3) (2012) 450-460.

[18] W.F. Louisos, D.L. Hitt, Viscous effects on performance of three-dimensional supersonic micronozzles, Journal of Spacecraft and Rockets, 49(1) (2012) 51-58.

[19] J.A. Potkay, The promise of microfluidic artificial lungs, Lab on a Chip - Miniaturisation for Chemistry and Biology, 14(21)(2014) 4122-4138.

[20] K.M. Kovach, M.A. Labarbera, M.C. Moyer, B.L. Cmolik, E. Van Lunteren, A. Sen Gupta, J.R. Capadona, J.A. Potkay, In vitro evaluation and in vivo demonstration of a biomimetic, hemocompatible, microfluidic artificial lung, Lab on a Chip -Miniaturisation for Chemistry and Biology, 15(5) (2015) 1366-1375.

[21] J. Jang, S.T. Wereley, Gaseous slip flow analysis of a micromachined flow sensor for ultra small flow applications, J. Micromech. Microengin., 17(2) (2007) 229-237.

[22] A. Vittoriosi, J.J. Brandner, R. Dittmeyer, A sensor-equipped microchannel system for the thermal characterization of rarefied gas flows, Experimental Thermal and Fluid Science, 41 (2012) 112-120.

[23] A. Demsis, B. Verma, S. V. Prabhu, A. Agrawal, Experimental determination of heat transfer coefficient in the slip regime and its anomalously low value, Phys. Review E, 80 (2009) 016311.

[24] A. Demsis, B. Verma, S. V. Prabhu, A. Agrawal, Heat transfer coefficient of gas flowing in a circular tube under rarefied condition. Int. J. Thermal Sciences, 49 (2010) 1994-1999.

[25] G. A. Bird, Molecular gas dynamics and the direct simulation of gas flows, Clarendon Press, Oxford, 1994.

[26] F. Sharipov, V. Seleznev, Data on internal rarefied gas flows, Journal of Physical and Chemical Reference Data, 27(3) (1998) 657-706.

[27] E. M. Sparrow, S. H. Lin, Laminar heat transfer in tubes under slip-flow conditions, J. Heat Transf., 84(4) (1962) 363-369.

[28] T. Ewart, P. Perrier, I.A. Graur, J.G. Méolans, Mass flow rate measurements in a microchannel, from hydrodynamic to near free molecular regimes, J. Fluid Mech., 584 (2007) $337-356$. 
[29] M. Gad-el Hak, Comments on "critical view on new results in micro-fluid mechanics", Int. J. Heat Mass Transf., 46(20) (2003) 941-3945.

[30] S. Colin, Rarefaction and compressibility effects on steady and transient gas flows in microchannels, Microfluidics and Nanofluidics, 1(3) (2005) 268-279.

[31] W.-M. Zhang, G. Meng, X. Wei, A review on slip models for gas microflows, Microfluidics and Nanofluidics, 13(6) (2012) 845-882.

[32] D.A. Lockerby, J.M. Reese, M.A. Gallis, Capturing the Knudsen layer in continuum- fluid models of nonequilibrium gas flows, AIAA Journal, 43(6) (2005) 1391-1393.

[33] D.A. Lockerby, J.M. Reese, On the modelling of isothermal gas flows at the microscale, J. Fluid Mech., 604 (2008) 235-261.

[34] L. O'Hare, D.A. Lockerby, J.M. Reese, D.R. Emerson, Near-wall effects in rarefied gas micro-flows: some modern hydrodynamic approaches, Int. J. Heat Fluid Flow, 28(1) (2007) $37-43$.

[35] L. O'Hare, T.J. Scanlon, D.R. Emerson, J.M. Reese, Evaluating constitutive scaling models for application to compressible microflows, Int. J. Heat Mass Transf., 51(5-6) (2008) 12811292.

[36] C. L. M. H. Navier, Mémoire sur les lois du mouvement des fluides, Mémoires de l'Académie Royale des Sciences de l'Institut de France, 6 (1823) 389-440.

[37] J. C. Maxwell, On stresses in rarefied gases arising from inequalities of temperature, Philosophical Transactions of the Royal Society of London, 170(6) (1879) 231-256.

[38] S. K. Loyalka, N. Petrellis, S. T. Stvorick, Some numerical results for the BGK model: thermal creep and viscous slip problems with arbitrary accommodation of the surface, Physics of Fluids, 18 (1975) 1094-1099.

[39] C. Cercignani, Mathematical methods in kinetic theory, Springer, Boston, 1990.

[40] D.A. Lockerby, J.M. Reese, D.R. Emerson, R.W. Barber, Velocity boundary condition at solid walls in rarefied gas calculations, Physical Review E - Statistical, Nonlinear, and Soft Matter Physics, 70(1) (2004) 017303.

[41] S. Colin, Gas microflows in the slip flow regime: A critical review on convective heat transfer, J. Heat Transf., 134(2) (2012) 020908.

[42] M. von Smoluchowski, Ueber wärmeleitung in verdünnten gasen, Annalen der Physik, 300(1) (1898) 101-130.

[43] H.P. Kavehpour, M. Faghri, Y. Asako, Effects of compressibility and rarefaction on gaseous flows in microchannels, Num. Heat Transf. A, 32(7) (1997) 677-696. 
[44] M. Miyamoto, W. Shi, Y. Katoh, J. Kurima, Chocked flow and heat transfer of low density gas in a narrow parallel-plate channel with uniformly heating walls, Int. J. Heat Mass Transf., 46 (2003) 2685-2693.

[45] C. Hong, Y. Asako, J.H. Lee, Heat transfer characteristics of gaseous flows in microchannel with constant heat flux. Int. J. Thermal Sciences, 46(11) (2007) 1153-1162.

[46] Z. Sun, Y. Jaluria. Convective heat transfer in pressure-driven nitrogen slip flows in long microchannels: The effects of pressure work and viscous dissipation, Int. J. Heat Mass Transf., 55(13-14) (2012) 3488-3497.

[47] N. G. Hadjiconstantinou, Dissipation in small gaseous flows, J. Heat Transf., 125 (2003) 944-947.

[48] C. Hong, Y. Asako, Some considerations on thermal boundary condition of slip flow, Int. J. Heat Mass Transf., 53(15-16) (2010) 3075-3079.

[49] Z. Sun, Y. Jaluria, Numerical modeling of pressure-driven nitrogen slip flow in long rectangular microchannels, Num. Heat Transf. A, 56(7) (2009) 541-562.

[50] A. Quasi Zade, M. Renksizbulut, J. Friedman, Heat transfer characteristics of developing gaseous slip-flow in rectangular microchannels with variable physical properties, Int. 1057 J. Heat Fluid Flow, 32(1) (2011) 117-127.

[51] Z. Sun, Y. Jaluria, Conjugate thermal transport in gas flow in long rectangular microchannel, Journal of Electronic Packaging, Transactions of the ASME, 133(2) (2011) 021008.

[52] S. E. Turner, L. C. Lam, Faghri M., O. J. Gregory, Experimental investigation of gas flow in microchannel, J. Heat Transf., 126 (2004) 753-763.

[53] Y. Ji, K. Yuan, J. N. Chung, Numerical simulation of wall roughness on gaseous flow and heat transfer in a microchannel, Int. J. Heat Mass Transf., 49 (2004) 1329-1339.

[54] G. Croce, P. D'Agaro, Compressibility and rarefaction effect on heat transfer in rough microchannels, Int. J. Thermal Sciences, 48 (2009) 252-260.

[55] S. Hossainpour, M. Hakak Khadem, Investigation of fluid flow and heat transfer characteristics of gases in microchannels with condsideration of different roughness shapes at slip flow regime, Nanoscale Microscale Thermophysical Engineering, 14 (2010) 137-151.

[56] O. Rovenskaya, G Croce, Numerical investigation of microflow over rough surfaces: coupling approach, J. Heat Transf., 135(1001005) (2013) 1-8.

[57] R.B. Bird, W.E. Stewart, E.N. Lightfoot, Transport phenomena, second ed., J. Wiley and Sons, Hoboken, 2002.

[58] S. H. Maslen, On heat transfer in slip flow, Journal of the Aeronautical Sciences, 25 (1958) 400-401. 
[59] W. Shi, M. Miyamoto, Y. Katoh, J. Kurima, Chocked flow of low density gas in a narrow parallel-plate channel with adiabatic walls, Int. J. Heat Mass Transf., 44 (2001) 2555-2565.

[60] K. Ramadan, I. Tlili, A Numerical Study of the Extended Graetz Problem in a Microchannel with Constant Wall Heat Flux: Shear Work Effects on Heat Transfer, Journal of Mechanics, 31 (2015) 733-743.

[61] K. Ramadan, Pressure Work and Viscous Dissipation Effects on Heat Transfer in a ParallelPlate Microchannel Gas Flow, Journal of Mechanics, (2017) 1-12.

[62] E.B. Arkilic, M.A. Schmidt, K.S. Breuer, Gaseous slip flow in long microchannels, J. Microelectromechanical Systems, 6(2) (1997) 167-178.

[63] C. Cai, Q. Sun, I. D. Boyd, Gas flows in microchannels and microtubes, J. Fluid Mech., 589 (2007) 305-314.

[64] O Touazi, E. Chénier, R. Eymard, Simulation of natural convection with the collocated clustered finite volume scheme, Computers and Fluids, 37 (2008) 1138-1147.

[65] E. Chénier, R. Eymard, R. Herbin, O. Touazi, Collocated finite volume schemes for the simulation of natural convective flows on unstructured meshes, Int. J. Num. Methods Fluids, 56(11) (2008) 2045-2068.

[66] E. Chénier, R. Eymard, O. Touazi, Numerical results using a collocated finite volume scheme on unstructured grids for incompressible fluid flows, Num. Heat Transf. B, 49(3) (2006) 259-276.

[67] C. Tchekiken, X. Nicolas, Beaudey-Laubier L.H., G. Lauriat, Simulation numérique d'écoulements anisothermes de gaz en micro canal : effets de compressibilité et de glissement, In 21ème Congrès Français de Mécanique, CFM2013, August 2013.

[68] C. Tchekiken, Modélisations et simulations numérique d'écoulements compressibles dans des micro-conduites, PhD thesis, Université Paris Est, 2014.

[69] C. Tchekiken, E. Chénier, X. Nicolas, G. Lauriat, A propos de la modélisation numérique de la convection de gaz en micro conduites, In Congrès Français de Thermique, SFT2014, number 8613, Lyon, France, June 2014.

[70] C. Tchekiken, E. Chénier, X. Nicolas, G. Lauriat, On the numerical modeling of compressible forced convection of gases in micro-channels, In Proceedings of the 5th 1099 International Conference on Heat Transfer and Fluid Flow in Microscale, HTFFM-V, number O-128-146, Marseille, France, April 2014.

[71] G.L. Morini, Y. Yang, H. Chalabi, M. Lorenzini, A critical review of the measurement techniques for the analysis of gas microflows through microchannels, Experimental Thermal and Fluid Science, 35(6) (2011) 849-865. 


\section{Nomenclature}

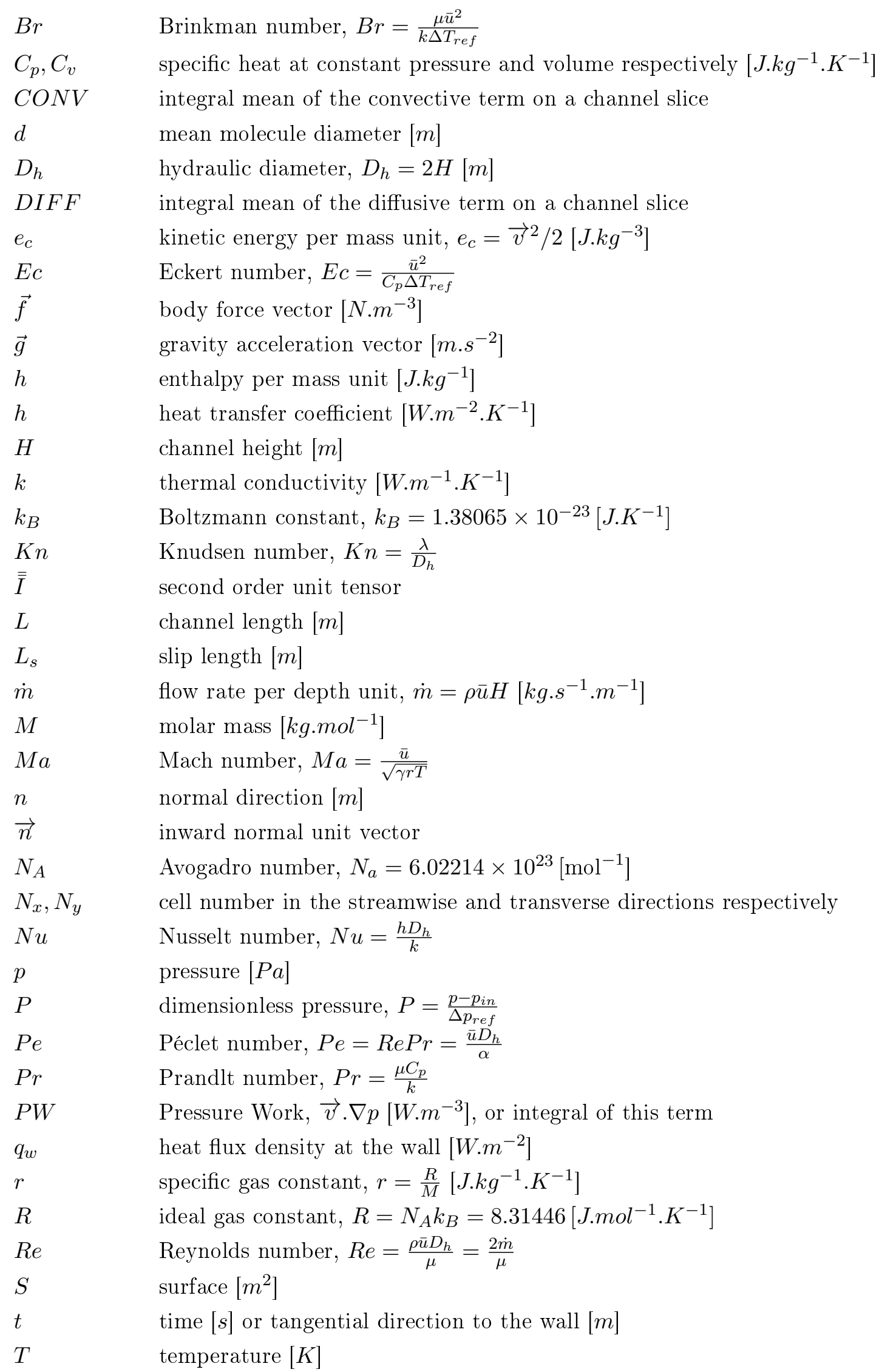


streamwise velocity component along $x$-direction $\left[\mathrm{m} . \mathrm{s}^{-1}\right]$

bulk streamwise velocity $\left[m . s^{-1}\right]$

reference velocity, $u_{\text {ref }}=\frac{\epsilon D_{h} \Delta p_{\text {ref }}}{\mu_{w}}\left[\mathrm{~m} . \mathrm{s}^{-1}\right]$

dimensionless streamwise velocity component, $U=u / \bar{u}$

transverse velocity component along $y$-direction $\left[\mathrm{m} . \mathrm{s}^{-1}\right]$

velocity vector

molecule speed $\left[{\left.\mathrm{m} . \mathrm{s}^{-1}\right]}^{-1}\right.$

dimensionless transverse velocity component, $V=v / \bar{u}$

Viscous Dissipation, $\overline{\bar{\tau}}: \nabla \vec{v}\left[W \cdot m^{-3}\right]$, or integral of this term

Viscous Stress Power at the wall, $\left.(\overline{\bar{\tau}} \cdot \vec{v}) \cdot \vec{n}\right|_{g}\left[W \cdot m^{-2}\right]$, or integral of this term streamwise coordinate $[\mathrm{m}]$

dimensionless streamwise coordinate, $X=x / L$

transverse coordinate $[\mathrm{m}]$

dimensionless transverse coordinate, $Y=y / D_{h}$

\section{Greek symbols}

$\alpha$

$\beta$

$\gamma$

$\delta \Omega, \delta \Sigma$

$\epsilon$

$\theta$

$\lambda$

$\mu$

$\nu$

$\xi_{T}$

$\rho$

$\sigma_{T}$

$\sigma_{u}$

$\sigma_{\mu}$

$\sigma_{\theta}$

$\overline{\bar{\tau}}$

$\Delta p_{\text {ref }}$

$\Delta T_{\text {ref }}$

$\Delta x$

$\Phi_{v}$

thermal diffusivity $\left[\mathrm{m}^{2} / \mathrm{s}\right]$

thermal expansion coefficient $\left[K^{-1}\right]$

isentropic coefficient, $\gamma=\frac{C_{p}}{C_{v}}$

volume and surface of a channel slice

small parameter of the asymptotic analysis, $\frac{D_{h}}{L}=2 \frac{H}{L}$

dimensionless temperature, $\theta=\frac{T-T_{w}}{\Delta T_{r e f}}$

mean free path, $\lambda=\frac{\mu}{p} \sqrt{\frac{\pi r T}{2}}[m]$

dynamic viscosity $[$ Pa.s]

kinematic viscosity $\left[m^{2} \cdot s^{-1}\right]$

temperature jump coefficient

gas density $\left[\mathrm{kg}^{-\mathrm{m}^{-3}}\right]$

thermal accommodation coefficient

Tangential Momentum Accommodation coefficient (TMAC)

viscous slip coefficient

thermal slip coefficient

shear stress tensor $\left[N . m^{-2}\right]$

reference pressure, difference between the inlet and outlet pressures, $\Delta p_{\text {ref }}=p_{\text {in }}-p_{\text {out }}[\mathrm{Pa}]$ reference temperature difference, $\Delta T_{\text {ref }}=\frac{\mu_{w} \bar{u}^{2}}{k_{w}}[K]$

space step in $x$-direction

dimensionless viscous dissipation

\section{Subscripts}

av

arithmetic average quantity

conv

relative to the convective term 


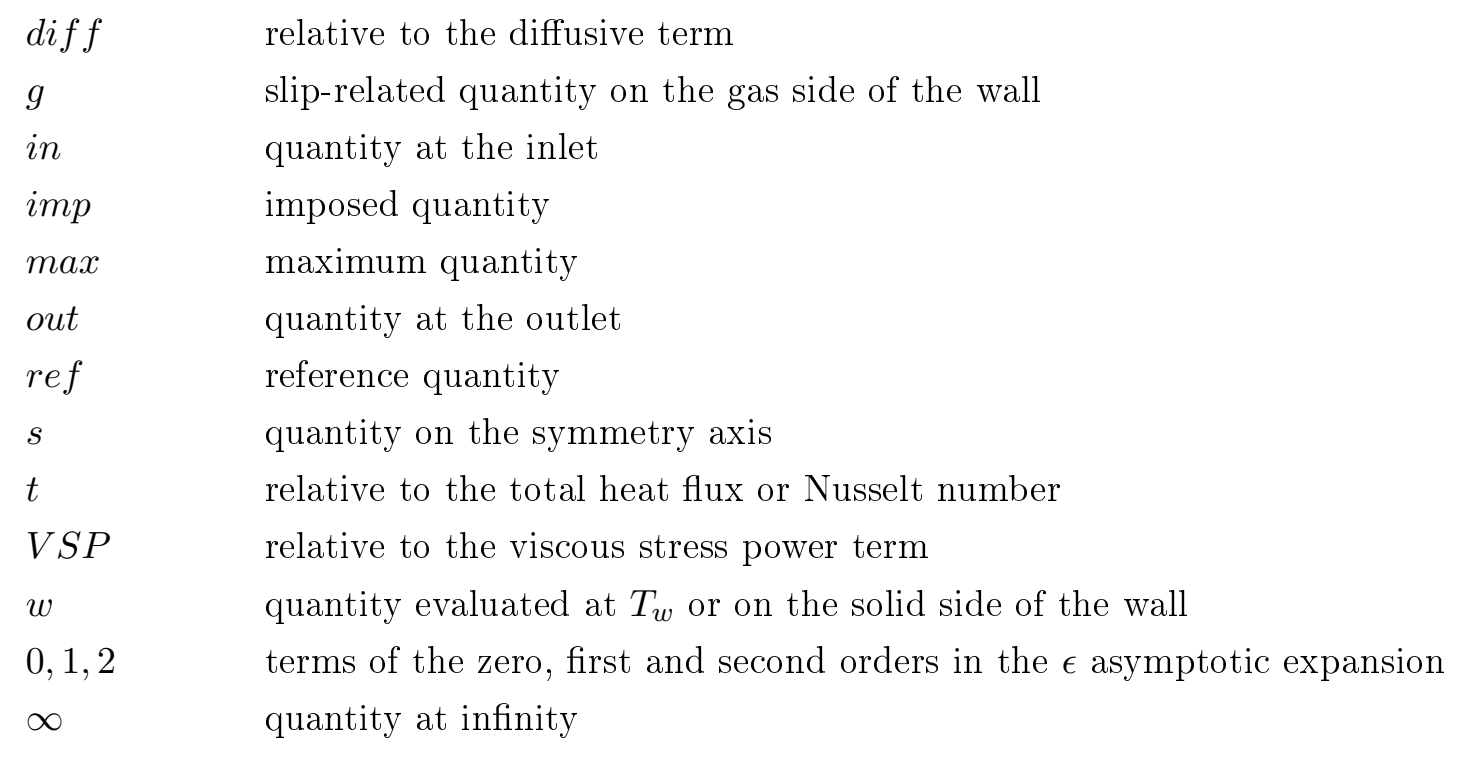

\section{Superscripts}

* dimensionless quantity

- $\quad$ bulk or integral average quantity 